\title{
Single-cell RNA sequencing reveals a dynamic stromal niche within the evolving tumour microenvironment
}

Sarah Davidson*2, Mirjana Efremova*1, Angela Riedel ${ }^{2}$, Bidesh Mahata ${ }^{1}$, Jhuma Pramanik ${ }^{1}$, Jani Huuhtanen ${ }^{3}$, Gozde Kar ${ }^{1}$, Roser Vento-Tormo ${ }^{1}$, Tzachi Hagai ${ }^{1}$, Xi Chen ${ }^{1}$, Muzlifah A. Haniffa $^{4}$, Jacqueline D. Shields ${ }^{\# 2}$, Sarah A. Teichmann ${ }^{\# 1}$

1 Wellcome Sanger Institute, Wellcome Genome Campus, Hinxton, Cambridge CB10 1SA, UK. ${ }^{2}$ Medical Research Council Cancer Cell Unit, Hutchison/Medical Research Council Research Centre, Cambridge, UK. ${ }^{3}$ Hematology Research Unit Helsinki, University of Helsinki, Finland. ${ }^{4}$ Institute of Cellular Medicine, Newcastle University, Newcastle upon Tyne, NE2 4HH, UK

*\# These authors contributed equally to this work

\# Corresponding authors js970@mrc-cu.cam.ac.uk and st9@sanger.ac.uk

\section{Summary}

Non-cancerous stromal cells represent a highly diverse compartment of the tumour, yet their role across tumour evolution remains unclear. We employed single-cell RNA sequencing to determine stromal adaptations in murine melanoma at different points of tumour development. Naive lymphocytes recruited from lymph nodes underwent activation and clonal expansion within the tumour, prior to PD1 and Lag3 expression, while tumourassociated myeloid cells promoted the formation of a suppressive niche through cytokine secretion and inhibitory $T$ cell interactions. We identified three temporally distinct cancerassociated fibroblast (CAF) populations displaying unique signatures, and verified these in human datasets. In early tumours, immune CXCL12/CSF1 and complement -expressing CAFs supported recruitment of macrophages, whereas contractile CAFs became more prevalent in later tumours. This study highlights the complex interplay and increasing diversity among cells that co-evolve with the tumour, indicating that from early stages of development, stromal cells acquire the capacity to modulate the immune landscape towards suppression. 


\section{Introduction}

To aid their growth and development, malignant cells cultivate a supporting niche of 'normal' cells, known as the tumour stroma. This niche comprises non-immune cells such as fibroblasts, blood and lymphatic endothelial cells, as well as numerous immune populations ${ }^{1}$. In particular, the balance of anti- vs. pro-tumour leukocytes can dictate tumour fate ${ }^{2,3}$, andin many cases suppressive populations persist to support immune escape and prevent tumour clearance. While, immunotherapies such as anti-CTLA4, anti-PD1 and anti-PD-L1 show efficacy in a large number of melanoma patients, a significant proportion do not respond to this treatment ${ }^{4-7}$. Thus, there remains a critical need to uncover novel therapeutic targets. The numerous mechanisms through which stromal cells promote tumour growth, represent a wealth of opportunities for therapeutic intervention. However, the evolving tumour microenvironment is extremely dynamic, continually adapting to both soluble and mechanical cues which induce significant heterogeneity within the stromal compartment ${ }^{8}$.

In particular, extensive heterogeneity has been reported within tumour fibroblast populations. Cancer associated fibroblasts (CAFs) are the most abundant non-immune stromal component, secreting growth factors, promoting angiogenesis, facilitating metastasis and regulating immune infiltrates ${ }^{9-15}$. Although they express typical fibroblast markers, such as fibroblast activation protein (FAP), platelet derived growth factor receptors $\alpha$ (PDGFRa) and $\beta$ (PDGFR $\beta$ ), podoplanin (PDPN), Thy- 1 and $\alpha$-smooth muscle actin (aSMA), no single marker universally identifies all CAFs within the tumour stroma ${ }^{16-18}$. To date, many studies rely on positive selection approaches, in which one or two markers are used to isolate CAFs for functional characterisation. Consequently, these findings likely reflect a sub-population of cells and may bias our perceptions of CAF function. It remains unclear whether fibroblast subpopulations with distinct roles are present in the tumour microenvironment.

Current approaches lack the resolution to visualise the true extent of stromal heterogeneity and may mask rare populations, or cellular phenotypes, that could be critical for tumour survival. Therefore, we have employed single-cell RNA sequencing (scRNAseq) to profile both immune and non-immune stromal populations from the B16-F10 murine model of melanoma. Furthermore, cells were isolated from both primary tumours and draining lymph nodes, at different stages of tumour development, enabling a systems level interrogation of the melanoma microenvironment in real-time. Here, we identified the presence of a diverse immune landscape, in which the composition and phenotype of leukocytes change as the tumour evolves. In particular, effector T cells displaying signs of dysfunction, were detected 
predominantly in late stage tumours. This work also highlighted significant heterogeneity within the CAF compartment of the primary tumour. Three distinct CAF populations were identified; immune, desmoplastic and contractile, each displaying unique functional and temporal characteristics key to the tumour. At early time points, the "immune" and 'desmoplastic' populations dominated, yet at later stages, the third 'contractile' subset became more prevalent. Using a unique database of known ligand-receptor interactions, we investigated communication between different stromal populations to reveal complex interplay between the 'immune' CAF subset, macrophages and T-cells, which ultimately contributed to T-cell dysfunction.

\section{Results}

\section{Identification of stromal populations within the developing tumour microenvironment}

Specific immune populations were enriched for, based on surface marker expression, and index sorted from tumours and lymph nodes at day 5, 8 and 11. Isolated single cells were then profiled using Smart-seq2 (Fig. 1a and Extended Data Fig. 1a). To avoid the biases associated with isolation of non-immune stroma, B16-F10 melanoma cells were injected into CAG-EGFP mice exhibiting widespread eGFP expression. This enabled a negative selection approach which did not rely upon expression of surface markers. Tumour and immune cells were removed by selecting $\mathrm{GFP}^{+} \mathrm{CD}^{-}$cells only, with the remaining stromal cells separated into two fractions, based on CD31 expression (Fig. 1a). CD $31^{+}$cells represented both blood and lymphatic endothelial cells, whereas CD31- cells were largely composed of fibroblasts. Graph-based clustering ${ }^{19}$ of more than 4600 cells that passed the computational quality control (see Methods and Extended Data Fig. 1b), confirmed that populations clustered together with the exception of endothelial cells from tumour and lymph node (Fig. 1b, c and Extended Data Fig. 2). Within each annotated cluster, we could also identify sitedetermined groupings, specific to either the tumour or lymph node derived cells (Fig. 1b). In particular T cells and dendritic cells exhibited differential clustering between tumour sites and lymph nodes. Moreover, in contrast to recent studies, our approach of sampling over multiple times points, at each site, enabled us to investigate temporal changes between sites and adaptation within each population (Fig. 1b).

\section{Dynamics of immune stroma}


We first sought to delineate relationships within the specific innate immune populations isolated from tumour-associated tissues. Clusters corresponding to macrophages/monocytes, natural killer cells (NK), plasmacytoid DCs (pDC) and conventional DCs (cDCs), were identified based on known markers (Macrophages/Monocytes, Adgre1(F4-80); FcyR1, NK Ncr1; pDCs, Bst2, Siglech; cDCs, Itgax (Cd11c); Fig. 2a, b, c and Supplementary Table 1). Moreover, multiple DC populations were observed that reflect the Cd11c+ cDC1 and Cd11c+Cd11b+(Itgam) cDC2 phenotypes. CDC1 and CDC2 titles were assigned based on expression of known markers including Clec9a, Baft3 (cDC1), Cd11b and Sirpa (cDC2) (Fig. 2 c,b). Two further clusters that lacked lineage markers for adaptive immune cells, as well as ILCs, yet express low Cd11b and Cd11c, were termed migratory DCs due to expression of the DC transcription factor Baft3 and upregulation of Ccr7.

Each DC population further separated according to their location in either the tumour or draining lymph node (Fig. 2a). Consistent with the steady state, tumour CDC1 cells expressed the dermal DC marker Cd103 (Itgae), whereas the LN population express Cd8a, a marker of lymph node resident $\mathrm{CDC} 1$ populations. Cd11b+ MPs in the LN consisted of Adgre1+, Ccr2+ macrophages, as well as a Cd11c+ resident CDC2 population (Fig. 2b). Investigation into transcriptional phenotypes of myeloid cells revealed that cells located in the tumour, compared to the lymph node were more activated, yet also displayed immunosuppressive properties. Within tumour MPs, no clear delineation between an M1 or the pro-tumour M2 phenotype was observed, yet chemokines involved in T cell recruitment and suppressive mediators such as Cd274 (PDL1), Arg1, Ido1, Adora2a/b were expressed. Furthermore, while tumour DC populations displayed increased expression and a wider variety of co-stimulatory molecules, than their lymph node counterparts, they lack expression of cytokines required to induce durable $T$ cell responses. This is particularly relevant in regard to $\mathrm{CDC1}$ cells, which can cross-present tumour antigen to cytotoxic T lymphocytes. Indeed, while both tumour and LN CDC1 cells upregulate genes involved in crosspresentation pathway, including components of the proteasome (Tap1, Tap and Sec61), expression of these genes was higher in the tumour subset (Extended Data Fig. 3d). Although this may indicate that tumour $\mathrm{CDC1}$ populations have increased potential to activate anti-tumour lymphocytes, tumour DCs also expressed immunosuppressive molecules such as Cd274 (PDL1), Pdcd1/g2 (PDL2) and Lgals9 (galectin-9), known to induce $\mathrm{T}$ cell exhaustion (Fig. 2d). Interestingly, across all myeloid populations, expression of immunosuppressive molecules increased at later time points, whereas co-stimulatory molecules were expressed consistently throughout tumour development. This indicates that 
tumour resident myeloid populations are present and activated at early stages of tumour growth, yet become more suppressive as the tumour progresses.

T cell populations from tumours and draining lymph nodes were transcriptionally distinct, clustering based upon their subtype, but also location (Fig. 3a). At the lymph node, T cells exhibited a more naive phenotype compared to those present at the primary tumour (Fig. 3b). While tumour resident CD4+ T cells were more activated, a significant proportion highly expressed Treg-associated genes at the tumour (Fig. 3b). Similarly, within the CD8+ T cell compartment, those at the tumour were also more activated, expressing high levels of Ifng (IFNy), Prf1 (perforin) and Gzmb (granzyme B). However, these cells were also less functional, as evident by expression of Pdcd1 (PD1), Lag3 and Tim3 (Fig. 3b). To identify transcriptional adaptations in CD8 $+\mathrm{T}$ cells, at different stages of tumour development, we performed pseudotime analysis that revealed a trajectory of gene expression associated with functional changes in these cells. This confirmed that the majority of $T$ cells within the lymph node were naive displaying high expression of Sell and Tcf7 (Fig. 3c and d). Arrival at the tumour corresponded with acquisition of activation signatures, including upregulation of Ifng (IFNy) and Gzmb (Granzyme B). Furthermore, T cell receptor sequence analysis identified clonal expansion (Fig. $3 \mathrm{c}$ and d) specifically within tumours at later time points. This was accompanied by expression of the proliferation marker Mki67 and exhaustion markers Pdcd1, Lag3 and Tim3 (Fig. 3c and d). Interestingly, a subset of the potentially exhausted $\mathrm{CD}^{+} \mathrm{T}$ cells also showed expression of Entpd1 (CD39), which was recently identified as a marker to distinguish tumour-specific and bystander $\mathrm{CD}^{+} \mathrm{T}$ cells ${ }^{20}$. Together, these results indicate that $T$ cell recruitment from the $L N$ is followed by activation and subsequent functional defects in situ. These functional defects correspond with the gain of immunosuppressive properties in myeloid populations at later time points, indicating that the immune stroma transitions from immunogenic to suppressive phenotypes.

\section{Non-immune stroma comprise three distinct functional populations}

As the non-immune stromal components are emerging as immune modulators, we also examined this compartment during tumour progression, focussing on the CAFs. Across all time points, we identified three distinct CAF populations referred to as CAF 1, 2 and 3 (Fig. 4a). As expected, expression of commonly used CAF markers was extremely variable across the fibroblasts (Fig. 4e and Extended Data Fig. 6a), yet, expression of specific marker combinations correlated with individual clusters. CAF1 could be distinguished from CAF3 by its high levels of Pdpn, Pdgfra and Cd34, while Acta2 (aSMA) was strongly expressed by the 
latter population. However, CAF2 represents an intermediate population that was $P d p n^{+}$ Pdgfra ${ }^{+}$and displayed low expression of Acta2 and Cd34 (Fig. 4e).

Importantly, each cluster displayed distinct functional signatures (Fig. 4f, Extended Data Fig. $5 \mathrm{c}, \mathrm{d})$, indicating that fibroblast populations may have specific roles within the tumour microenvironment. CAF $1\left(\mathrm{Pdpn}^{+} \mathrm{Pdgfra}^{+} \mathrm{Cd} 34^{\text {high }}\right)$ upregulated genes involved recruitment and regulation of immune cells, including the cytokines $C x c / 12, C s f 1$ and Cc/8, cytokine receptors I/6ra and I/6st, as well as components of the complement cascade C3, C2 and C4b. Thus, CAF1 may engage in immune cross-talk. In contrast, CAF2 $\left(P d p n^{+} P_{\text {dgfra }}{ }^{+}\right.$ $C d 34^{\text {low }}$ ) expressed genes encoding extracellular matrix (ECM) components including numerous collagen family members and Postn and Tnc. These ECM components are strongly associated with a fibrotic matrix, a feature common to developed tumours ${ }^{21}$. Thus, it is possible that this CAF population drives the desmoplastic reaction associated with tumour development. CAF3 $\left(\right.$ Acta2 $\left.{ }^{\text {high }}\right)$ was enriched for genes involved in regulation and rearrangement of the actin cytoskeleton. In particular, this cluster upregulated Rock1, Mlc2 and Mlck, which are responsible for the contraction of actin stress fibres. Thus, CAF 3 likely represents a more contractile fibroblast subset. CAF3 also expressed some pericyteassociated markers such as Cspg4 (Ng2), Mcam and Rgs5 (Extended Data Fig. 7a), leading us to consider whether this population may contain pericytes. The same markers were also observed in the Pdpn+ fibroblasts (FRCs) in the lymph nodes however, (Extended Data Fig. $7 \mathrm{~b}$ ) indicating that, and consistent with previous reports, their expression is not limited to pericytes. Thus, to identify whether CAF3 represent a pericyte or fibroblast population, we examined expression of aSMA, $\mathrm{Ng} 2$ and Mcam in relation to the endothelial marker CD31. While these were observed surrounding vessels in adjacent skin, they were rarely associated with intratumoural vessels, but could be detected in peritumoural spindle-shaped cells distinct from the vasculature (Extended Data Fig. $7 \mathrm{c}-\mathrm{e}$ ).

Our approach highlighted the dynamic nature of CAF populations within a developing tumour. Although each CAF population was detected throughout the time course, different clusters dominated at specific time points. Early day 5 tumours were primarily comprised of fibroblasts from the Pdpn ${ }^{+}$Pdgfra ${ }^{+}$CAF1 and 2, whereas the Acta2 ${ }^{\text {high }}$ CAF3 population was largely restricted to later stages, implying a selective enrichment in developed tumours. This enrichment may be supported, in part, by our observation of proliferation specifically within CAF2 and 3 (Fig. 4b). Proliferation of a subset of CAFs, within the tumour microenvironment, was confirmed by incorporation of the thymidine analogue, EdU (Extended Data Fig. 6c). Moreover, the majority of both mouse skin and human skin fibroblasts resembled the cells from CAF1 (Fig. 4e). Together, this data illustrates that the CAF compartment and its 
associated functions are dynamic, adapting to localised cues and the changing needs of an evolving tumour.

To validate the existence of these different populations in the tumour microenvironment, we first confirmed each subset based on their unique marker repertoire. Consistent with sequencing data, confocal imaging revealed that CAF markers PDPN and PDGFRa largely colocalised, while expression of aSMA was more distinct (Fig. 5a). The 'immune' CAF1 marker CD34, colocalized with both PDPN and PDGFRa, indicating the presence of a CD34 ${ }^{\text {high }}$ subpopulation. Furthermore, a distinct $C D 34^{\text {high }} \alpha S M A^{\text {low }}$ CAF subset could be clearly distinguished (Fig. 5a). Although the RNA level, PDPN ${ }^{+} \mathrm{PDGFRa}^{+}$CD34 ${ }^{\text {high }} \mathrm{CAFs}$ are aSMA- (Extended Data Fig. 6a), we observed some colocalization between these four markers at the protein level. This may represent the intermediate PDPN ${ }^{+}$PDGFRa $^{+}$CAF2 population, which also expressed low levels of CD34 and aSMA.

To examine the inflammatory phenotype associated with the CAF1 population in more detail, we focused on two highly expressed immune modulatory factors, CXCL12 and CSF1. Using flow cytometry we identified each population using their differential expression of CD34 and aSMA. Tumour fibroblasts were identified using multiple CAF markers, after exclusion of other stromal populations (Extended Data Fig. 8a), and divided into CD34 ${ }^{\text {high }}$ aSMA $^{\text {low }}(\mathrm{CAF} 1), \mathrm{CD} 34^{\text {low }}$ aSMA ${ }^{\text {low }}(\mathrm{CAF} 2)$ and CD34 ${ }^{\text {low }} \alpha S M A^{\text {high }}$ (CAF3) subsets. Reflecting our sequencing data, this confirmed CXCL12 expression was highest in the CAF1 subset, followed by intermediate expression in CAF2 and low expression in CAF3 (Fig. 5b), this was further verified with RNAscope showing localization of CXCL12 and CD34 ${ }^{+}$at the RNA level in situ (Extended Data Fig. 6d). Confocal imaging also confirmed CD34 ${ }^{+}$CAFs as a source of CSF1 in the tumour stroma both at the protein (Fig. 5c) and RNA levels (Extended Data Fig. 6d). Having validated the presence of these functionally distinct populations, we next evaluated their prevalence at different stages of tumour development (Fig. 5e). This showed that, as a percentage of the total CAF population, the proportion of of CD34 ${ }^{\text {low }}$ aSMA ${ }^{\text {high }}$ CAF3 subset was greater at day 11 compared to day 5. This data supports our earlier proposal of a dynamic fibroblast niche, which evolves alongside its malignant tumour core.

Together, these data have identified the presence of distinct CAF populations that dynamically co-evolve with the tumour to support its changing requirements (Fig. $5 \mathrm{~g}$ ) and indicate that CAFs acquire the capacity to influence the tumour immune landscape from early stages of development. 


\section{Cross-talk between 'immune'CAFs in infiltrating myeloid cells}

Next, we sought to elucidate the potential functional consequences of specific stromal populations to the ensuing immune response. Thus we focused on the early CAF1 "immune" population and examined the cross-talk with responsive immune populations recruited to the tumour. To systematically study interactions within the tumour microenvironment, we predicted cell-cell communication networks based on CellPhoneDB, a manually curated repository of ligands, receptors and their interactions integrated with a statistical framework to infer cell-cell communication networks from single cell transcriptomic data (Vento-Tormo, Efremova et al, Nature, in press). This approach highlighted likely interactions involved in angiogenesis, immune cell recruitment and immune modulation between stromal populations in the tumour (Fig. 6a, Supplementary Table 4).

We identified CAF-immune interactions, for example between C3/CXCL12/CSF1-expressing CAFs enriched in early stages of tumour development, and macrophages positive for CXCR4, CSFR1, C3AR1 respectively (Fig. 6 a, b). IF imaging of tumour sections allowed us to correlate single cell data with location in situ. Indeed, at the protein level, both CSFR1 ${ }^{+}$ and $\mathrm{CXCR} 4^{+}$macrophages were detected in close contact with CD34 ${ }^{\text {high }}$ fibroblasts in the tumour stroma (Fig. 6c). The combination of transcriptome profiling and cell-cell communication pipeline enabled us to assign these immune interactions specifically to the CAF1/2 subpopulations. Further chemokine-receptor interacting pairs, identified as statistically significant, occured between the immune CAF1 subpopulation, myeloid, Treg and $\mathrm{CD}^{+} \mathrm{T}$ cells (Fig. 6a). The recruited macrophages exhibited the capacity to both attract T cells, via specific cytokine-receptor signals such as CXCL10, and suppress their function through the PDL1-PD1 axis (Fig. 6a and d). Our approach highlighted additional cell-cell communications enriched between tumour infiltrating immune such as recruitment of NK cells through $\mathrm{cDC} 1$-cell derived chemokines receptors $\mathrm{XCR} 1^{22}$. Additionally, we found that the Tregs express high levels of Nt5e (CD73) and Entpd1 (CD39, Fig. 6a and Fig. 3b), which act together to convert ATP to adenosine who release has been shown to dampen the immune system ${ }^{23}$. The adenosine receptors Adora2a and Adora2b were found upregulated on the migratory DCs and the macrophages respectively.

Collectively, these findings provide new insights into the complex interplay among cells within the evolving tumour microenvironment (Fig. 6d), where multiple immunosuppressive mechanisms coexist within an increasingly heterogeneous stromal compartment. 


\section{Discussion}

It is becoming increasingly evident that non-malignant stromal cells such as endothelial cells, fibroblasts, and infiltrating immune cells found within a tumour, provide significant and varied supporting roles as disease progresses. The heterogeneity and dynamic nature of the tumour microenvironment can make identification of the roles of the different stromal components challenging. The emergence of scRNA-seq has enabled new insights into tumour biology not detectable by previous methods, and has been key to reveal the true degree intratumoural heterogeneity ${ }^{24,25}$.

In this study, we used a single-cell transcriptomic approach to characterise the stromal landscape within the evolving microenvironment. Our scRNA-seq analysis revealed the gradual development of a suppressive immune microenvironment and defined fibroblast subsets with distinct functional signatures. This approach also highlighted the complexity of cross-talk between the different stromal components as a tumour evolves.

Both clinical studies and the success of immune checkpoint inhibitors have emphasised the importance of the immune system, particularly T cells and macrophages, in deciding tumour fate or response to therapy. While clinical studies have repeatedly demonstrated the presence of exhausted $T$ cells with poor prognosis ${ }^{2,3}$, the steps leading to this point and sites of activation are less clear. Here we showed distinct gene profiles between sites, with lymph nodes acting as a source of naive T cells. Once at the tumour, pseudotime analysis illustrated the trajectory of $\mathrm{T}$ cell development within the evolving tumour microenvironment from naive, through clonal expansion and activation (enriched granzyme and IFN expression) phases, to upregulation of exhaustion markers in late tumours (PD1 and Lag3).

A diverse repertoires of myeloid cells were observed within the tumour, and similar to T cells, tumour myeloid populations were more activated than in the lymph node displaying high levels of phagocytosis, antigen presenting and co-stimulatory associated genes. Once at the tumour however, an increase in the level of suppressive factors produced, likely in response to local cues, was detected. Many of these cues served to act upon infiltrating $\mathrm{T}$ cell populations to confound the suppressive environment already developing. Moreover, our dynamics data indicates that inhibitory signalling commenced in later phases of tumour development, coinciding with the emergence of $T$ cell dysfunction.

While infiltrating immune populations can have a profound effect on tumour fate, a growing body of evidence indicates that CAFs play a supporting role in the tumour 
microenvironment ${ }^{26}$. To date, few studies have investigated whether fibroblast phenotypes change as the tumour develops. Our analysis revealed the existence of three CAF subsets which possess unique characteristics and temporal dynamics, indicative of distinct, specialized roles. Based on their transcriptional signatures, these populations were termed "immune", "desmoplastic" and "contractile". The 'immune' CAF1 population, which was detected from early stages of tumour development, upregulated cytokines CSF1 and CXCL12, as well as complement components $\mathrm{C} 3$ and $\mathrm{C} 4 \mathrm{~b}$, which are known to recruit and regulate immune cells. Conversely, the desmoplastic population upregulated ECM components and may be responsible for the production of a fibrotic matrix. Finally, the third population, dominated at later stages of tumour development in well-established lesions, expressed genes involved in the contraction of actin stress fibres, indicating a contractile phenotype.

It is likely that the alteration in population dynamics is induced by concomitant changes in the developing tumour. New environmental factors, such as nutrient availability and hypoxia arise, transforming the secretion profile of malignant cells. Furthermore, changes in the phenotype and function of the immune stroma, may add to the local cytokine milieu. Biophysical cues also likely contribute to adaptation of CAF populations. It has been reported that matrix rigidity is critical for the maintenance of CAF phenotypes ${ }^{34}$, and that mechanical and soluble cues are required for the induction of aSMA expression ${ }^{35,36}$. Thus it is likely that the combination of a stiff matrix, produced by 'desmoplastic' CAFs, and cytokine exposure, may upregulate aSMA expression and expansion of 'contractile' CAFs in later tumours. No single commonly applied marker identified all CAF subsets. Instead the 3 populations were distinguished based on their discrete expression of CAF marker combinations. While both 'immune' and 'desmoplastic' CAFs express PDPN and PDGFRa, the 'immune' population was distinguished based on its high expression of CD34. The 'contractile' population is largely negative for these markers and instead expresses aSMA.

Interestingly, and consistent with other studies, this population also shared some common markers with pericytes, such as $\mathrm{Ng} 2$, Mcam and Rgs $5^{37}$. While this could imply pericyte contamination, CAF3 also produced matrix components such as Col1a1, Col1a2 (collagen1) Fn1 (fibronectin1) and Sparc. Although expression of these genes was lower in CAF3 compared with the desmoplastic CAF2 population, production of these proteins is associated with a fibroblast phenotype. Pericytes are located on the surface of blood vessels where they provide structural support, as well as regulating endothelial cell phenotypes. Like fibroblasts, these cells represent a heterogeneous population that is difficult to distinguish by a specific marker. While markers such as NG2 and RGS5 are often used to identify pericytes, their 
expression is context-dependent, varying between tissues and during pathology ${ }^{38}$. Furthermore, many markers, such as aSMA, PDGFR $\beta, \mathrm{Ng} 2$ and Mcam are shared by both pericytes and activated myofibroblasts, making it difficult to distinguish one from the other 39,40 . This was reflected in our data, in which expression of multiple pericyte markers was observed in the CAF3 population as well as in PDPN+ lymph node FRCs. A similar phenomenon was reported in a subcutaneous model of breast cancer, in which expression of pericyte markers was also observed in FRC populations ${ }^{41}$. Thus, the most robust method to differentiate between these cell types is to assess whether they are associated with endothelial vessels. In our melanoma model, we observed aSMA, NG2 and MCAM positive cells both associated with vessels and in more peripheral locations. However, the number of vessel associated pericytes was very small, as the majority of vessels were composed of endothelial cells alone. Consequently, CAF3 may embody a mixed population of mesenchymal cells, containing both pericytes and fibroblasts, that share similar surface marker expression and functional properties. Interestingly, the close relationship between these two cell types has led to the suggestion that pericytes may differentiate into activated myofibroblasts during pathology. In both liver and kidney fibrosis, as well as in tumour models, upon the initiation of fibrosis or during growth of malignant cells, pericytes dissociate from the endothelium and begin to express the markers such as aSMA and produce collagen ${ }^{42-45}$. Therefore, it is possible that fibroblasts within CAF3 may arise from pericyte origins. Overall, this data highlights the limitations of using single marker approaches to isolate and characterise mesenchymal cells, which can lead to contamination and selection bias.

Importantly, and relevant to the clinic, our murine data was mirrored in the setting of human melanoma, with shared patterns of CAF marker expression ${ }^{24}$. Here, CAFs expressing PDPN, PDGFR $\alpha$ and CD34 also clustered together, whereas those expressing aSMA were more distinct. Furthermore, these PDPN ${ }^{+} P D G F \alpha^{+} C D 34^{+}$CAFs displayed high expression of CXCL12. Other immunomodulators such as PD-1 ligands and, in particular, complement components were observed in both systems suggesting that the immune function of these populations are a conserved feature and retained in human melanoma. Although the CAF $1 / 2$ clusters were not as distinct as the mouse model, the cohort of CAFs was much smaller. This discrepancy can be explained by the fact that many of these patients had received immunotherapies prior to resection ${ }^{24}$, and in light of the immunomodulatory capacity of these clusters, we cannot rule out an effect of treatment on the wider stromal landscape.

Our findings also compliment recent investigations into CAF heterogeneity in a range of different solid cancers, each reporting a distinct $\alpha S_{M A}^{+}$fibroblast phenotype ${ }^{25,46,47}$. 
Moreover, aSMA fibroblasts highly expressing ECM components, similar to our 'desmoplastic' population have been described in both colorectal and head and neck cancers ${ }^{25,46}$. Furthermore, in a preclinical model of pancreatic ductal adenocarcinoma (PDAC), another aSMA- CAF population was shown to display an inflammatory profile ${ }^{47}$. Similarly, a subpopulation of CXCL12-secreting fibroblasts was reported in human breast cancer. However, in contrast to our results, a proportion of this CAF population was aSMA+ 48. This suggests that the three populations we have identified may be a universal feature of the tumour stroma in a variety of cancer types. Subtle difference between the fibroblast populations reported, such as marker expression and the range of cytokines produced, likely reflect the local milieu of soluble and mechanical cues, as well as environmental pressures unique to the tumour type.

Sequencing of paired immune and non-immune stroma provided the opportunity to investigate signalling between different stromal compartments. Using a recently reported database of receptor-ligand interactions, we were able to infer cross-talk between the 'immune' CAF subset and Cd11 $b^{+} C d 11 c^{-}$macrophages, via the CXCL12-CXCR4,CSF1CSF1R and C3-C3ar1 axes. While both of CXCL12 and CSF1 are reported to recruit macrophages to the tumour stroma and induce a suppressive phenotype, the role of the complement cascade in the tumour microenvironment is more ambiguous 29,49-52. Complement components are typically thought to aid immune clearance by increasing opsonization and phagocytosis, formation of the membrane attack complex and recruitment of multiple immune populations ${ }^{53}$. However, in a malignant context, the complement cascade has been shown to promote tumorigenesis and induce immune suppression. In particular, complement components $\mathrm{C} 3 \mathrm{a}$ and $\mathrm{C} 5 \mathrm{a}$ and their cognate receptors C3AR1 and C5AR1 are linked with recruitment of suppressive myeloid cells and T cell dysfunction ${ }^{54-58}$. Interestingly, the CAF1 subset represented the greatest source of C3 in the primary tumour. This component acts upstream within the complement cascade, inducing the cleavage and activation of downstream factors. Thus, while CAF1 secreted C3 may recruit macrophages to the tumour stroma, C3 activation of complement components, such as C5a, may broadly suppress immune function.

Thus, 'immune' CAFs may aid in the recruitment and polarisation of suppressive macrophage populations, which then further contribute to the development of immune privilege by suppressing $T$ cell function. Furthermore, the CAF1 subpopulation may directly induce $T$ cell dysfunction by activation of the complement cascade. This additional layer of immune regulation by CAFs is also consistent with recent studies in pre-clinical models of PDAC and melanoma, which suggested that blockade of either CXCL12-CCR4, CSF1- 
CSF1R or complement receptors, act to synergise with checkpoint inhibitors $57,32,59$. With inhibitors targeting both CXCR4 and CSF1R, as well as numerous components of the complement cascade, currently in clinical trials (for example, CXCR4: AMD3100 and PF06747143, CSF1R: JNJ-40346527 and PLX5622, C3: APL-1 APL-2 ), enrichment of 'immune' CAFs may highlight patients that would benefit from this treatment, in combination with checkpoint immunotherapies.

In summary, we have demonstrated the power of scRNAseq to define the tumour stromal landscape, highlighting the dynamic and adaptive nature of both immune and non-immune stroma within an evolving tumour microenvironment, and revealedpotential cross-talk between these two compartments. We identified 3 CAF clusters with distinct functional and temporal features; the immune subset supporting recruitment and induction of an immunosuppressive macrophage phenotype providing an alternative, indirect mechanism to dampen $\mathrm{T}$ cell mediated anti-tumour immunity.

\section{References}

1. Turley, S. J., Cremasco, V. \& Astarita, J. L. Immunological hallmarks of stromal cells in the tumour microenvironment. Nat. Rev. Immunol. 15, 669-682 (2015).

2. Galon, J. et al. Type, density, and location of immune cells within human colorectal tumors predict clinical outcome. Science 313, 1960-1964 (2006).

3. Sato, E. et al. Intraepithelial CD8+ tumor-infiltrating lymphocytes and a high $\mathrm{CD} 8+/$ regulatory $\mathrm{T}$ cell ratio are associated with favorable prognosis in ovarian cancer. Proc. Natl. Acad. Sci. U. S. A. 102, 18538-18543 (2005).

4. Hamid, O. et al. Safety and Tumor Responses with Lambrolizumab (Anti-PD-1) in Melanoma. N. Engl. J. Med. 369, 134-144 (2013).

5. Topalian, S. L. et al. Safety, activity, and immune correlates of anti-PD-1 antibody in cancer. N. Engl. J. Med. 366, 2443-2454 (2012).

6. Brahmer, J. R. et al. Safety and activity of anti-PD-L1 antibody in patients with advanced cancer. N. Engl. J. Med. 366, 2455-2465 (2012).

7. Hodi, F. S. et al. Improved survival with ipilimumab in patients with metastatic 
melanoma. N. Engl. J. Med. 363, 711-723 (2010).

8. Junttila, M. R. \& de Sauvage, F. J. Influence of tumour micro-environment heterogeneity on therapeutic response. Nature 501, 346-354 (2013).

9. Jia, C.-C. et al. Cancer-associated fibroblasts from hepatocellular carcinoma promote malignant cell proliferation by HGF secretion. PLoS One 8, e63243 (2013).

10. Orimo, A. et al. Stromal fibroblasts present in invasive human breast carcinomas promote tumor growth and angiogenesis through elevated SDF-1/CXCL12 secretion. Cell 121, 335-348 (2005).

11. Guo, X., Oshima, H., Kitmura, T., Taketo, M. M. \& Oshima, M. Stromal fibroblasts activated by tumor cells promote angiogenesis in mouse gastric cancer. J. Biol. Chem. 283, 19864-19871 (2008).

12. Dumont, N. et al. Breast fibroblasts modulate early dissemination, tumorigenesis, and metastasis through alteration of extracellular matrix characteristics. Neoplasia 15, 249 262 (2013).

13. Gaggioli, C. et al. Fibroblast-led collective invasion of carcinoma cells with differing roles for RhoGTPases in leading and following cells. Nat. Cell Biol. 9, 1392-1400 (2007).

14. Calon, A. et al. Dependency of colorectal cancer on a TGF- $\beta$-driven program in stromal cells for metastasis initiation. Cancer Cell 22, 571-584 (2012).

15. Harper, J. \& Sainson, R. C. A. Regulation of the anti-tumour immune response by cancer-associated fibroblasts. Semin. Cancer Biol. 25, 69-77 (2014).

16. Cortez, E., Roswall, P. \& Pietras, K. Functional subsets of mesenchymal cell types in the tumor microenvironment. Semin. Cancer Biol. 25, 3-9 (2014).

17. Augsten, M. Cancer-associated fibroblasts as another polarized cell type of the tumor microenvironment. Front. Oncol. 4, 62 (2014).

18. Roswall, P. \& Pietras, K. Of mice and men: a comparative study of cancer-associated fibroblasts in mammary carcinoma. Ups. J. Med. Sci. 117, 196-201 (2012).

19. Satija, R., Farrell, J. A., Gennert, D., Schier, A. F. \& Regev, A. Spatial reconstruction of single-cell gene expression data. Nat. Biotechnol. 33, 495-502 (2015). 
20. Simoni, Y. et al. Bystander CD8+ T cells are abundant and phenotypically distinct in human tumour infiltrates. Nature 557, 575-579 (2018).

21. Bonnans, C., Chou, J. \& Werb, Z. Remodelling the extracellular matrix in development and disease. Nat. Rev. Mol. Cell Biol. 15, 786-801 (2014).

22. Böttcher, J. P. et al. NK Cells Stimulate Recruitment of cDC1 into the Tumor Microenvironment Promoting Cancer Immune Control. Cell 172, 1022-1037.e14 (2018).

23. Vijayan, D., Young, A., Teng, M. W. L. \& Smyth, M. J. Targeting immunosuppressive adenosine in cancer. Nat. Rev. Cancer 17, 709 (2017).

24. Tirosh, I. et al. Dissecting the multicellular ecosystem of metastatic melanoma by singlecell RNA-seq. Science 352, 189-196 (2016).

25. Puram, S. V. et al. Single-Cell Transcriptomic Analysis of Primary and Metastatic Tumor Ecosystems in Head and Neck Cancer. Cell 171, 1611-1624.e24 (2017).

26. Kalluri, R. The biology and function of fibroblasts in cancer. Nat. Rev. Cancer 16, 582598 (2016).

27. Pickup, M. W. et al. Stromally derived lysyl oxidase promotes metastasis of transforming growth factor- $\beta$-deficient mouse mammary carcinomas. Cancer Res. 73, 5336-5346 (2013).

28. Hlatky, L., Tsionou, C., Hahnfeldt, P. \& Coleman, C. N. Mammary fibroblasts may influence breast tumor angiogenesis via hypoxia-induced vascular endothelial growth factor up-regulation and protein expression. Cancer Res. 54, 6083-6086 (1994).

29. Comito, G. et al. Cancer-associated fibroblasts and M2-polarized macrophages synergize during prostate carcinoma progression. Oncogene 33, 2423-2431 (2014).

30. De Monte, L. et al. Intratumor T helper type 2 cell infiltrate correlates with cancerassociated fibroblast thymic stromal lymphopoietin production and reduced survival in pancreatic cancer. J. Exp. Med. 208, 469-478 (2011).

31. Li, T. et al. Hepatocellular carcinoma-associated fibroblasts trigger NK cell dysfunction via PGE2 and IDO. Cancer Lett. 318, 154-161 (2012).

32. Feig, C. et al. Targeting CXCL12 from FAP-expressing carcinoma-associated fibroblasts 
synergizes with anti-PD-L1 immunotherapy in pancreatic cancer. Proc. Natl. Acad. Sci. U. S. A. 110, 20212-20217 (2013).

33. Özdemir, B. C. et al. Depletion of Carcinoma-Associated Fibroblasts and Fibrosis Induces Immunosuppression and Accelerates Pancreas Cancer with Reduced Survival. Cancer Cell 28, 831-833 (2015).

34. Calvo, F. et al. Mechanotransduction and YAP-dependent matrix remodelling is required for the generation and maintenance of cancer-associated fibroblasts. Nat. Cell Biol. 15, 637-646 (2013).

35. Arora, P. D., Narani, N. \& McCulloch, C. A. The compliance of collagen gels regulates transforming growth factor-beta induction of alpha-smooth muscle actin in fibroblasts. Am. J. Pathol. 154, 871-882 (1999).

36. Li, Z. et al. Transforming growth factor-beta and substrate stiffness regulate portal fibroblast activation in culture. Hepatology 46, 1246-1256 (2007).

37. Lambrechts, D. et al. Phenotype molding of stromal cells in the lung tumor microenvironment. Nat. Med. 24, 1277-1289 (2018).

38. Thomas, H., Cowin, A. J. \& Mills, S. J. The Importance of Pericytes in Healing: Wounds and other Pathologies. Int. J. Mol. Sci. 18, (2017).

39. Sugimoto, H., Mundel, T. M., Kieran, M. W. \& Kalluri, R. Identification of fibroblast heterogeneity in the tumor microenvironment. Cancer Biol. Ther. 5, 1640-1646 (2006).

40. Zheng, B. et al. CD146 attenuation in cancer-associated fibroblasts promotes pancreatic cancer progression. Mol. Carcinog. 55, 1560-1572 (2016).

41. Cremasco, V. et al. FAP delineates heterogeneous and functionally divergent stromal cells in immune-excluded breast tumors. Cancer Immunol Res (2018). doi:10.1158/2326-6066.CIR-18-0098

42. Mederacke, I. et al. Fate tracing reveals hepatic stellate cells as dominant contributors to liver fibrosis independent of its aetiology. Nat. Commun. 4, 2823 (2013).

43. Chen, Y.-T. et al. Platelet-derived growth factor receptor signaling activates pericytemyofibroblast transition in obstructive and post-ischemic kidney fibrosis. Kidney Int. 80, 
1170-1181 (2011).

44. Lin, S.-L., Kisseleva, T., Brenner, D. A. \& Duffield, J. S. Pericytes and perivascular fibroblasts are the primary source of collagen-producing cells in obstructive fibrosis of the kidney. Am. J. Pathol. 173, 1617-1627 (2008).

45. Hosaka, K. et al. Pericyte-fibroblast transition promotes tumor growth and metastasis. Proc. Natl. Acad. Sci. U. S. A. 201608384 (2016).

46. $\mathrm{Li}, \mathrm{H}$. et al. Reference component analysis of single-cell transcriptomes elucidates cellular heterogeneity in human colorectal tumors. Nat. Genet. 49, 708-718 (2017).

47. Öhlund, D. et al. Distinct populations of inflammatory fibroblasts and myofibroblasts in pancreatic cancer. J. Exp. Med. 214, 579-596 (2017).

48. Costa, A. et al. Fibroblast Heterogeneity and Immunosuppressive Environment in Human Breast Cancer. Cancer Cell 33, 463-479.e10 (2018).

49. Pixley, F. J. Macrophage Migration and Its Regulation by CSF-1. Int. J. Cell Biol. 2012, 501962 (2012).

50. Priceman, S. J. et al. Targeting distinct tumor-infiltrating myeloid cells by inhibiting CSF1 receptor: combating tumor evasion of antiangiogenic therapy. Blood 115, 1461-1471 (2010).

51. Fleetwood, A. J., Lawrence, T., Hamilton, J. A. \& Cook, A. D. Granulocyte-macrophage colony-stimulating factor (CSF) and macrophage CSF-dependent macrophage phenotypes display differences in cytokine profiles and transcription factor activities: implications for CSF blockade in inflammation. J. Immunol. 178, 5245-5252 (2007).

52. Pyonteck, S. M. et al. CSF-1R inhibition alters macrophage polarization and blocks glioma progression. Nat. Med. 19, 1264-1272 (2013).

53. Reis, E. S., Mastellos, D. C., Ricklin, D., Mantovani, A. \& Lambris, J. D. Complement in cancer: untangling an intricate relationship. Nat. Rev. Immunol. 18, 5-18 (2018).

54. Markiewski, M. M. et al. Modulation of the antitumor immune response by complement. Nat. Immunol. 9, 1225-1235 (2008).

55. Gunn, L. et al. Opposing roles for complement component C5a in tumor progression 
and the tumor microenvironment. J. Immunol. 189, 2985-2994 (2012).

56. Vadrevu, S. K. et al. Complement c5a receptor facilitates cancer metastasis by altering T-cell responses in the metastatic niche. Cancer Res. 74, 3454-3465 (2014).

57. Nabizadeh, J. A. et al. The Complement C3a Receptor Contributes to Melanoma Tumorigenesis by Inhibiting Neutrophil and CD4+ T Cell Responses. J. Immunol. 196, 4783-4792 (2016).

58. Wang, Y. et al. Autocrine Complement Inhibits IL10-Dependent T-cell-Mediated Antitumor Immunity to Promote Tumor Progression. Cancer Discov. 6, 1022-1035 (2016).

59. Zhu, Y. et al. CSF1/CSF1R blockade reprograms tumor-infiltrating macrophages and improves response to T-cell checkpoint immunotherapy in pancreatic cancer models. Cancer Res. 74, 5057-5069 (2014).

60. Picelli, S. et al. Full-length RNA-seq from single cells using Smart-seq2. Nat. Protoc. 9, 171-181 (2014).

61. Patro, R., Duggal, G., Love, M. I., Irizarry, R. A. \& Kingsford, C. Salmon provides fast and bias-aware quantification of transcript expression. Nat. Methods 14, 417-419 (2017).

62. Qiu, X. et al. Reversed graph embedding resolves complex single-cell trajectories. Nat. Methods 14, 979-982 (2017).

63. Stubbington, M. J. T. et al. T cell fate and clonality inference from single-cell transcriptomes. Nat. Methods 13, 329-332 (2016).

64. Scialdone, A. et al. Computational assignment of cell-cycle stage from single-cell transcriptome data. Methods 85, 54-61 (2015).

65. Zheng, G. X. Y. et al. Massively parallel digital transcriptional profiling of single cells. Nat. Commun. 8, 14049 (2017).

66. Hagai, T. et al. Gene expression variability across cells and species shapes innate immunity. Nature (2018). doi:10.1038/s41586-018-0657-2 


\section{Materials and Methods}

\section{Mouse models}

Animals were housed in accordance with UK regulations and experiments were performed under project licences PPL 80/2574 or PPL P8837835. The C57BL/6 derived B16.F10 melanoma cell line was purchased from American Type Culture Collection (ATCC) and cultured in Dulbecco's Modified Eagle medium (DMEM, Life Technologies), supplemented with $1 \%$ Penstrep and $10 \%$ FBS. $2.5 \times 10^{5}$ B16 cells were injected, subcutaneously, into the shoulders of either wild type (WT) C57BL/6 mice, or C57BL/6-Tg(CAGEGFP)1310sb/LeySopJ mice (Jackson Laboratory). After 5, 8 and 11 days animals were sacrificed and tissues collected for analysis. In addition, skin was also taken from nontumour bearing mice.

\section{Tissue Processing}

Tumours were mechanically dissociated and digested in $1 \mathrm{mg} / \mathrm{ml}$ collagenase $D$ (Roche), $1 \mathrm{mg} / \mathrm{ml}$ collagenase $A$ (Roche) and $0.4 \mathrm{mg} / \mathrm{ml}$ DNase (Roche) in PBS, at $37^{\circ} \mathrm{C}$ for $2 \mathrm{hs}$. Lymph nodes were mechanically dissociated and digested with $1 \mathrm{mg} / \mathrm{ml}$ collagenase $A$ (Roche) and $0.4 \mathrm{mg} / \mathrm{ml}$ DNase (Roche) in PBS, at $37^{\circ} \mathrm{C}$. After $30 \mathrm{mins}$, Collagenase D (Roche) was added (final concentration of $1 \mathrm{mg} / \mathrm{ml}$ ) to lymph node samples and digestion was continued for a further 30 mins. EDTA was added to all samples to neutralise collagenase activity (final concentration $(5 \mathrm{mM})$ and digested tissues were passed through $70 \mu \mathrm{m}$ filters (Flacon) ready for staining.

\section{Isolation of Single Cells}

Single cells were isolated from processed tissues using fluorescence-activated cell sorting (FACS). Once processed, samples were incubated with a fixable fluorescent viability stain (Life Technologies) for 20mins (diluted 1:1000 in PBS) prior to incubation with conjugated primary antibodies for 30 mins at $4^{\circ} \mathrm{C}$. Antibodies were diluted in PBS $0.5 \%$ BSA according to table SX. Stained samples were index sorted, using the BD influx flow cytometer system, Single-cells were sorted in $2 \mu$ l of Lysis Buffer (1:20 solution of RNase Inhibitor (Clontech, 
cat. no. $2313 \mathrm{~A}$ ) in $0.2 \% \mathrm{v} / \mathrm{v}$ Triton X-100 (Sigma-Aldrich, cat. no. T9284)) in 96 well plates, spun down and immediately frozen at -80 degrees.

\section{Preparation of cDNA and sequencing}

Reverse transcription and cDNA pre-amplification were performed according to the SmartSeq2 protocol ${ }^{60}$ to obtain mRNA libraries from single-cells. Oligo-dT primer, dNTPs (ThermoFisher, cat. no. 10319879) and an ERCC RNA Spike-In Mix (1:50,000,000 final dilution, Ambion, cat. no. 4456740) were then added. Reverse Transcription and PCR were performed as previously published ${ }^{60}$, using $50 \mathrm{U}$ of SMARTScribe ${ }^{\mathrm{TM}}$ Reverse Transcriptase (Clontech, cat. no. 639538). cDNA libraries were prepared using the Nextera XT DNA Sample Preparation Kit (Illumina, cat. no. FC-131-1096), according to the protocol supplied by Fluidigm (PN 100-5950 B1). Single cell libraries were pooled, purified using AMPure XP beads (Beckman Coulter) and sequenced on an Illumina HiSeq 2500 aiming for and average depth of 1 Million reads/cell (paired-end 100-bp reads).

\section{Single-cell RNA sequencing analysis}

The SmartSeq2 data was quantified with Salmon ${ }^{61}$ (version 0.8.2), using the GENCODE mouse protein-coding transcript sequences. Transcript Per Million (TPM) values reported by Salmon were used for the quality control of the samples. In order to get the endogenous TPM values, we removed the ERCC's from the expression table and scaled the TPM's so that they sum to a million. Cells with less than 1500 detected genes and for which the total mitochondrial expression exceeded $20 \%$ were excluded from further analysis. Genes that were expressed in less than 3 cells were also removed.

Downstream analysis such as, clustering based on SNN graph-based clustering, differential expression analysis and visualisation were performed using the Seurat package ${ }^{19}$ (version 2.3.4) implemented in R. Clusters were identified using the community identification algorithm as implemented in the Seurat "FindClusters" function. Differential expression analysis was performed based on the Wilcoxon rank sum test. Clusters were annotated using canonical cell type markers. Two clusters of $\mathrm{dDC} 2$ in the tumour represented the same cell type and were therefore merged.

Trajectory modelling and pseudotemporal ordering of cells was performed with the Monocle 2 R-package $^{62}$ (version 2.8.0). The most highly variable genes were used for ordering the cells. Potential doublets and contaminating melanocytes and keratinocytes were excluded. 
We also removed a cluster for which the top markers were genes associated with dissociation-induced effects.

To further identify subpopulations, we reanalysed the $T$ cells, innate immune cells (myeloid and NK) and the CAFs separately, using the same workflow as described above. To account for the cell cycle heterogeneity in the T cell subsets. a cell cycle score was calculated for each cell and this score was then regressed out. We used the function "AddModuleScore" from Seurat and the list of G2M associated genes from Scaldoen et al. to calculate a cell cycle score for each cell.

Gene Set Enrichment Analysis (GSEA) (software.broadinstitute.org/gsea/index.jsp) was performed on genes that were differentially expressed between clusters, with a $p$ value < 0.05. Overlap with canonical GO categories (CP:BIOCARTA, CP:KEGG, CP:REACTOME) was assessed and the False Discovery Rate (FDR) calculated.

\section{T-cell receptor (TCR) analysis}

The TCR sequences for each single $T$ cell were assembled using $\operatorname{TraCeR}^{63}$ which allowed the reconstruction of the TCRs from scRNA-seq data and their expression abundance (transcripts per million, TPM), as well as identification of the size, diversity and lineage relation of clonal subpopulations. In total, we detected 77 TCR sequences with at least one paired productive $\alpha \beta$ or gamma-delta chain. Cells for which more than two recombinants were identified were excluded from further analysis.

\section{Cell cycle analysis}

The pair-based prediction method described by Scialdone et $a l^{64}$. and implemented in the $\mathrm{R}$ package scran was used to assign each cell a cell cycle stage. Briefly, using a training data, pairs of marker genes are identified such that the expression of the first gene in the training data is greater than the second in certain cell cycle stage but less than the second in all other stages. For each cell then, the method calculates the proportion of all marker pairs where the expression of the first gene is greater than the second in the test data.

\section{Putative interactions between cell types}

To enable a systematic analysis of cell-cell communication, we used CellPhoneDB (VentoTormo, Efremova et al., Nature, in press). CellPhoneDB is a manual curated repository of 
ligands, receptors and their interactions, integrated with a new statistical framework for inferring cell-cell communication networks from single cell transcriptome data. Briefly, in order to identify the most relevant interactions between cell types, we looked for the cell-type specific interactions between ligands and receptors. Only receptors and ligands expressed in more than $10 \%$ of the cells in the specific cluster were considered. We performed pairwise comparisons between all cell types. First, we randomly permuted the cluster labels of all cells 1000 times and determined the mean of the average receptor expression level of a cluster and the average ligand expression level of the interacting cluster. For each receptorligand pair in each pairwise comparison between two cell types, this generated a null distribution. By calculating the proportion of the means which are "as or more extreme" than the actual mean, we obtained a $p$-value for the likelihood of cell type-specificity of a given receptor-ligand complex. We then prioritized interactions that are highly enriched between cell types based on the number of significant pairs and manually selected biologically relevant ones. For the multi-subunit heteromeric complexes, we required that all subunits of the complex are expressed (using a threshold of 10\%), and therefore we used the member of the complex with the minimum average expression to perform the random shuffling.

\section{Mouse skin fibroblasts from healthy mice}

Skin samples from two 8-week old female C57BL/6 mice were processed, first by mechanical processing, followed by $2 \mathrm{~h}$ incubation with $0.5 \%$ collagenase $\mathrm{B}$ (Roche; 11088815001). Cells were then counted and loaded on the 10x Chromium machine. Libraries were prepared following the Chromium Single Cell 3' v2 Reagent Kit Manual ${ }^{65}$. Libraries were sequenced on an Illumina HiSeq 4000 instrument with 26 bp for read 1 and $98 \mathrm{bp}$ for read 2.

Droplet-based sequencing data was aligned, filtered and quantified using the Cell Ranger Single-Cell Software Suite (version 2.2.0), against the mouse reference genome provided by Cell Ranger. The data was analysed using the pipeline described above. Only the clusters identified as fibroblasts (based on expression of Col1a1, Col1a2) were considered for comparison with the CAF clusters.

\section{Human skin fibroblasts}

scRNA-seq data was downloaded from ArrayExpress (E-MTAB-6831) ${ }^{66}$. CD45-negative cells from a digested skin sample were taken from a human female and processed in a 10X Chromium machine (10X Genomics). Droplet-based sequencing data was aligned, filtered and quantified using the Cell Ranger Single-Cell Software Suite (version 1.2.0), against the 
GRCh38 human reference genome provided by Cell Ranger. The data was analysed using the pipeline described above. Only the clusters identified as fibroblasts (based on expression of COL1A1, COL1A2) were considered for comparison with the CAF clusters.

\section{Comparison of human and mouse skin fibroblasts with CAFs}

To compare the mouse and human skin fibroblasts with the CAFs, a logistic regression with L2-norm regularization and a multinomial learning approach (implemented by the scikit-learn function LogisticRegression) was trained on the CAF clusters, using the log-transformed normalized data. The model was used to predict the probabilities of each mouse and human skin cell belonging to each one of the CAF clusters (implemented by the predict_proba function).

\section{Flow Cytometry}

Following a $20 \mathrm{~min}$ incubation with a fixable fluorescent viability stain (see isolation of single cells), cells were incubated with primary antibodies, against cell surface markers, for $30 \mathrm{mins}$ at $4^{\circ} \mathrm{C}$. All primary antibodies were diluted according to table 1 in PBS $0.5 \%$ BSA. If required, fluorescently labelled streptavidin, diluted 1:300 in PBS 0.5\%BSA, was added for a further 30mins. To stain for intracellular targets samples were fixed and permeabilized using the FOXP3 kit (eBioscence), according to manufacturer's instructions. Fixation and permeabilization was only performed once staining for surface markers was completed. For investigation of CXCL12 expression, samples were incubated with Brefeldin-A (BFA, Biolegend) prior to the staining process. Samples were incubated with BFA (1:1000 dilution) both during tissue processing and for a further $4 \mathrm{hs}$ in 10\% FBS supplemented DMEM media (Life Technologies). Once staining was completed, samples were analysed using the BD LSR-Fortessa system.

\begin{tabular}{|c|c|c|c|c|}
\hline Target & Species & Company & Clone & Dilution \\
\hline CD45 APC-Cy7 & Rat & Biolegend & $30-F 11$ & $1: 300$ \\
\hline CD45 FITC & Rat & Biolegend & $30-F 11$ & $1: 300$ \\
\hline CD31 PE-Cy7 & Rat & eBioscience & 390 & $1: 300$ \\
\hline
\end{tabular}




\begin{tabular}{|c|c|c|c|c|}
\hline PDPN APC & Syrian Hamster & Biolegend & 8.1 .1 & $1: 300$ \\
\hline CD3e 488 & $\begin{array}{l}\text { Armenian } \\
\text { Hamster }\end{array}$ & Biolegend & $145-2 \mathrm{C} 1$ & $1: 300$ \\
\hline CD3e PE & $\begin{array}{l}\text { Armenian } \\
\text { Hamster }\end{array}$ & Biolegend & $145-2 C 1$ & $1: 300$ \\
\hline CD4 PE-Cy7 & Rat & eBioscience & GK1.5 & $1: 300$ \\
\hline CD8 780 & Rat & eBioscience & $53-6.7$ & $1: 300$ \\
\hline CD8 PE & Rat & eBioscience & $53-6.7$ & $1: 300$ \\
\hline Lag3 Biotin & Rat & Biolegend & C9B7W & $1: 300$ \\
\hline PD1 & Rat & Biolegend & RMP1-30 & $1: 300$ \\
\hline Ki67 & Rat & Biolegened & $16 A 8$ & $1: 100$ \\
\hline IL-7Ra APC & Rat & Biolegend & A7R34 & $1: 300$ \\
\hline B220 488 & Rat & Biolegend & RA3-6B2 & $1: 300$ \\
\hline CD11b 647 & Rat & Biolegend & $\mathrm{M} 1 / 70$ & $1: 300$ \\
\hline CD11c PE-Cy7 & $\begin{array}{c}\text { Armenian } \\
\text { Hamster }\end{array}$ & Biolegend & N418 & $1: 300$ \\
\hline aSMA & Mouse & $\begin{array}{l}\text { Thermo } \\
\text { Fisher }\end{array}$ & $1 \mathrm{~A} 4$ & $1: 200$ \\
\hline PDGFRa Biotin & Rat & Biolegend & APA5 & $1: 300$ \\
\hline PDGFRb Biotin & Rat & Biolegend & APB5 & $1: 300$ \\
\hline
\end{tabular}




\begin{tabular}{|c|c|c|c|c|} 
Thy1 APC-Cy7 & Rat & Biolegend & 30-H12 & $1: 300$ \\
\hline CD34 APC & $\begin{array}{l}\text { Armenian } \\
\text { Hamster }\end{array}$ & Biolegend & HM34 & $1: 200$ \\
\hline CXCL12 PE & Mouse & R\&D Systems & MAB350 & $1: 100$ \\
\hline
\end{tabular}

Table 1. Conjugated Antibodies

\section{Immunofluorescence}

Collected tissues were embedded in OCT medium (VWR) and snap frozen on dry ice. Frozen tissues were sectioned into $10 \mu \mathrm{m}$ slices and stored at $-80^{\circ} \mathrm{C}$. Sections were air dried and fixed in 50:50 acetone (Fluka): methanol (Fisher), at $-20^{\circ} \mathrm{C}$ for $2 \mathrm{mins}$ or $4 \%$ paraformaldehyde (PFA) for 10 minutes. If fixed with PFA, samples were permeabilized with $0.1 \%$ Triton for a further 10 minutes. After blocking for $1 \mathrm{~h}$ at room temperature (RT) with blocking buffer (10\% chicken serum and $2 \%$ Bovine Serum Albumin) in PBS, primary antibodies were applied overnight at $4^{\circ} \mathrm{C}$ or RT for $3 \mathrm{hs}$. To visualise samples, secondary antibodies (Life Technologies), conjugated to either Alexa Fluor 488, 594 or 647, or fluorescently labelled streptavidin, were added for $2 \mathrm{hs}$ at RT. Samples were incubated with the nuclear stain 4',6-diamidino-2-phenylindole (DAPI) for $10 \mathrm{mins}$ at $1 \mu \mathrm{g} / \mathrm{ml}$, before mounting with ProLong Gold (ThermoFisher) liquid mountant. Streptavidin and secondary antibodies were diluted 1:300 in blocking buffer and primary antibodies were diluted in blocking buffer according to Table 2. Confocal imaging was performed using the Zeiss LSM 880 microscope and processed using the Zeiss Blue software.

\begin{tabular}{|c|c|c|c|c|}
\hline Target & Species & Company & Clone & Dilution \\
\hline PDPN & Syrian Hamster & Biolegend & 8.1 .1 & $1: 100$ \\
\hline aSMA & Rabbit & abcam & Polyclonal & $1: 50$ \\
\hline PDGFRa & Goat & R\&D Systems & Polyclonal & $1: 50$ \\
& & & & \\
\hline
\end{tabular}




\begin{tabular}{|c|c|c|c|c|}
\hline CD34 & Rat & eBioscience & RAM34 & $1: 50$ \\
\hline F4/80 & Rat & AbDserotech & A3-1 & $1: 100$ \\
\hline F4/80 488 & Rat & AbDserotech & A3-1 & $1: 20$ \\
\hline CXCR4 & Rat & R \& D Systems & 247506 & $1: 50$ \\
\hline CSFR1 & Sheep & R \& D Systems & Polyclonal & $1: 50$ \\
\hline CXCL12 PE & Mouse & R \& D Systems & MAB350 & $1: 50$ \\
\hline CSF1 & Rabbit & ABGENT & Polyclonal & $1: 50$ \\
\hline NG2 & Rabbit & abcam & Polyclonal & $1: 50$ \\
\hline CD31 & Rat & Biolegend & MEC13.3 & $1: 100$ \\
\hline
\end{tabular}

\section{Table 2. Purified antibodies}

\section{EdU Incorporation}

B16 melanomas were established in wt C57BL/6 mice as previously stated. Tumours were collected after 11 days and frozen in OCT medium for histology. Intraperitoneal injections of $500 \mu \mathrm{g} / \mathrm{ml}$ of 5-ethynyl-2'-deoxyuridine (EdU) were performed every $24 \mathrm{hs}, 4$ days prior to culling. Sections were fixed at $-20^{\circ} \mathrm{C}$, in a mixture of acetone and methanol (50:50). After fixation, the EdU Click-it Alexa Fluor 647 kit (Invitrogen) was used to visualise incorporated EdU, according to the manufacturer's protocol. Following the click-it reaction, antibody staining was performed as previously stated.

\section{Data availability}

The raw sequencing data for the melanoma model has been deposited in ArrayExpress (experiment E-MTAB-7427) and the count table can be downloaded from https://www.ebi.ac.uk/gxa/sc/experiments/E-EHCA-2/Results. The mouse skin data from healthy mice was deposited in ArrayExpress (experiment E-MTAB-7417).

\section{Funding}


This project was supported by CRUK Cancer Immunology fund (Ref. 20193), ERC grants (ThSWITCH, grant number 260507; ThDEFINE, Project ID 646794), an EU FET-OPEN grant (MRG-GRAMMAR No 664918), and Wellcome Sanger core funding (No WT206194).

Acknowledgments. We thank all members of Teichmann and Shields Lab for helpful discussions.

Author contributions. S.A.T, J.D.S and B.M conceived the study. S.D, A.R, B.M and J.P performed the mouse experiments, sample/library preparation and histology staining. M.E and S.D analysed the data and interpreted the results with contributions from J.H and G.K. T.H and X.C performed the sample/library preparation for the healthy mouse skin samples. S.D, M.E, J.D.S and S.A.T wrote the manuscript. S.A.T and J.D.S co-directed the study. All authors read and accepted the manuscript.

Competing Interests: None declared.

\section{Main Figures}

Figure 1. Distinction of melanoma stromal populations with single-cell RNA-seq. a, Overview of experimental and sequencing workflow. b, tSNE visualisation of all cells sequenced with each cell colour coded for; cell type (left), site of origin (middle), time (right). c, Expression of marker genes for each cell type. NK, natural killer; migDC, migratory DC; DC LN, lymph node dendritic cell, cDC1/2, conventional dendritic cell; pDC, plasmacytoid DC; MAIT, Mucosal-associated invariant T cell; Endo lymph, lymphatic endothelial cell; Endo tumour, tumour endothelial cells; endo LN, lymph node endothelium; fibroblast LN, lymph node fibroblast.

Figure 2. Myeloid cell clusters in the tumour exhibit suppressive characteristics. a, tSNE plot of individual myeloid cells colored by site (tumour, dark grey; lymph node, light grey) and clusters marked by coloured lines. b, Violin plots showing expression of selected surface marker genes within each cell cluster displayed as Log $(T P M+1)$. c, tSNE plots showing expression of selected marker genes for macrophages and inflammatory and resident monocytes. d, Heatmap showing mean expression ( $\log (T P M+1))$ of co-stimulatory and suppressive genes for the identified cell clusters. e, Heatmap showing relative expression (z-score) of co-stimulatory and suppressive genes in all innate immune cells over time. f, Schematic diagram of the costimulatory and inhibitory receptors/ligands expressed 
on distinct myeloid subpopulations. migDC, migratory DC; DC LN, lymph node dendritic cell, cDC1/2, conventional dendritic cell; pDC, plasmacytoid DC; MP, mononuclear phagocyte.

Figure 3. T cells recruited from lymph nodes are activated in situ. a, tSNE plot of individual T cells colored by site (tumour, dark grey; lymph node, light grey) and annotated subpopulations marked by coloured lines. b, Heatmap showing relative expression (z-score) of functional gene groups for cell clusters. c, Pseudotime analysis of CD8+ T cell gene trajectories coloured by site (left), clonal expansion (middle) and tumour stage (days, right), arrow indicates time direction. d, Expression of activation-associated genes along the inferred pseudotime coloured by site; lymph node (green), Tumour (blue).

Figure 4. Distinct fibroblast clusters identified in melanoma tumours. a, tSNE plot of sequenced fibroblasts from tumours coloured by their associated cluster (left) or by tumour time point (right) b, tSNE visualisation of the proliferation marker Mki67 in the CAFs. c, bar plot depicting the ratio of CAF populations at each time point examined where the size of each coloured bar is proportional to percentage of total CAFs each population represents. Data presented as mean \pm SEM. * $\mathrm{P}<0.05$ (Two way anova with Tukey post-hok test). d, Heatmap showing average expression (Log(TPM+1)) of typical CAF markers. e, Heatmap depicting logistic regression analysis of normal mouse skin, indicating which of the $3 \mathrm{CAF}$ clusters these cells are most similar. $\mathbf{f}$, Heatmap of gene ontology pathways for differentially expressed genes in each cluster; cytokine-chemokine receptor interactions, complement cascade, Extracellular matrix interactions and actin cytoskeleton. Columns represent individual cells, rows display z scores.

Figure 5. Fibroblast subtypes in murine are mirrored in human disease a, Representative confocal images of PDPN, PDGFRa and aSMA in combination (left panel) or CD34 in combination with either PDPN PDGFRa or aSMA (right panel) in day 5 and day 11 tumours. Dashed line indicates the tumour border. Scale bars $100 \mu \mathrm{m}$. b, Verification of populations by flow cytometry. Representative plots depicting the method by which each population was distinguished, based on CD34 and aSMA expression (left panel) and a histogram showing intracellular CXCL12 expression in each population (middle). Fold change in mean fluorescence of normalised to the CD34 ${ }^{\text {high }}$ aSMA ${ }^{\text {low }}$ population at day 11 (right), is also shown. c, Representative confocal image of CSF1 expression in aSMAlow CD34+ CAF populations in day 5 and day 11 tumours. Scale bars 20 um. d, Flow cytometry quantification of the proportion of each CAF population at day 5 and day 11 tumours, displayed as as the percentage of the total CAF population. e, Heatmap displays expression ( $z$ scores, blue to red) of key markers and cytokines across CAFs clusters identified in 
human melanoma. f, Schematic diagram of the three CAF subpopulations. (a, c, d representative image from at least $n=3$ independent mice). (b) $n=12$ independent mice. (d) Day 5: $n=9$ tumours from 8 independent mice, Day 11: $n=11$ independent mice. Data presented as mean \pm SEM. ${ }^{*} \mathrm{P}<0.05$ as calculated using either a one way anova with Dunnett's post-hok test (b) or Student's T-test (d)

Figure 6. Stromal crosstalk supports the development of an immune suppressive niche. a, Overview of selected statistically significant specific interactions between stromal subsets and other cell types using a cell-cell communication pipeline based on CellPhoneDB. Size indicates $p$-values and colour indicates the means of the receptor/ligand pairs between two clusters. b, Violin plots displaying expression of ligands Cxcl12, Csf1, C3 and PDL1 and cognate receptors Cxcr4, Csf1r, C3ar1 and PD1 on respective stromal populations. c, Confocal images of representative tumour-tissue borders. CSFR1 or CXCR4 expressing macrophages located proximally to CD34+ CAFs (green, F4/80; red, CXCR4 or CSF1R; white, podoplanin; blue CD34. Scale bars, 20um. d, Schematic diagram of the dynamic cross-talk identified within the tumour microenvironment.

\section{Extended Data Figures}

Extended Data Figure 1. Gating strategy and quality control of SS2 data. a, Gating strategy for index sorted cell populations. b, Quality control of the scRNA-seq dataset. Histograms show distribution of the cells from all cells that passed the computational quality control ordered by number of detected genes and mitochondrial gene expression content.

Extended Data Figure 2. Marker genes. a, Heatmap showing relative expression (z-score) of the top 5 markers for each cluster presented in Fig. 1B.

Extended Data Figure 3. Overview of the innate subpopulations. a, tSNE plots visualisation of expression of $\mathrm{MHC}$ class I and II genes in the innate immune subpopulations. b, Violin plots showing expression of selected cytokines for each innate immune subpopulation, known to induce T-cell responses. c, Heatmap of gene ontology pathways for differentially expressed genes in innate immune subpopulations; Antigen presentation and cytokine-chemokine receptor interactions. Columns represent individual cells, rows display z scores. d, Bar plot (-log FDR) depicting the top 20 gene ontology pathways upregulated in $\mathrm{CDC} 1$ cells, located in the tumour compared to the lymph node 
(left). Heat map depicting genes in the Class $1 \mathrm{MHC}$ mediated antigen presentation pathway (right). Columns represent individual cells, rows display z scores.

Extended Data Figure 4. Macrophage markers. a, tSNE plots showing expression of M1 and M2 macrophage markers in the innate immune subpopulations.

Extended Data Figure 5. a, Percentage of $\mathrm{CD}^{+} \mathrm{T}$ cells (displayed as percentage of total $\mathrm{T}$ cells) and percentage of $\mathrm{Ki} 67^{+}, \mathrm{Lag}^{+}$and $\mathrm{PD} 1^{+}$, $\mathrm{T}$ cells (displayed as a percentage of total $\mathrm{CD}^{+} \mathrm{T}$ cells), in tumours and lymph nodes isolated from skin, day 5 and day 11 tumour bearing or control mice. Data presented as mean \pm SEM. ${ }^{*} \mathrm{P}<0.05$ as calculated using either a one way anova with Tukey post-hok test, $n=4$ independent mice per time point.

\section{Extended Data Figure 6. Distinct CAF subpopulations identified in the melanoma} mouse model. a, tSNE plots showing expression of typical CAF markers. b, Bar plot (-log FDR) depicting the top 50 gene ontology pathways upregulated in each CAF population. C, IF imaging of EdU incorporation in a subset of CAFs in day 11 tumours. Scale bars $50 \mu \mathrm{m}$ (c, d, Representative confocal images of CSF1 and CXCL12 mRNA transcripts in CD34+ PDGFRa+ fibroblasts of day 11 tumours following RNAscope in situ hybridization technology. Scale bars 20um.

Extended Data Figure 7. a, Heatmap showing expression of canonical fibroblasts and pericytes markers in the CAFs. b, tSNE plots of all sequenced cells, showing expression of typical pericyte markers is also detected in PDPN ${ }^{+}$lymph node fibroblasts. c and d, IF imaging showing $\alpha \mathrm{SMA}^{+}$and $\mathrm{NG}^{+}$cells both distinct from (top panel) and associated with (middle panel) $\mathrm{CD} 31^{+}$blood vessels. Bottom panels show an abundance of $\mathrm{CD} 31^{+}$blood vessels that are not surrounded by pericytes.

Extended Data Figure 8. CAF validation and human clustering. a, Gating strategy for flow cytometry characterization of CAFs. $\mathbf{b}$, tSNE plots showing expression of CAF marker genes in the human melanoma dataset ${ }^{24}$.

\section{Supplementary Tables}


Supplementary table 1. Differentially expressed genes amongst the innate immune system clusters. This table provides the differentially expressed genes between the innate immune subpopulations ( $q$-value $<0.1)$.

Supplementary table 2. Pseudotime analysis of the CD8 T cells. Genes identified as varying significantly along the CD8 T cells trajectory ( $q$-value $<0.1)$.

\section{Supplementary table 3. Differentially expressed genes amongst the three CAF} clusters. This table provides the differentially expressed genes between the three CAF subpopulations ( $q$-value<0.1).

Supplementary table 4. List of interactions in the tumour infiltrating cells. This table provides the list of interaction pairs between clusters in Fig. 1B resulting from our cell-cell communication pipeline. Sheet 1 - $p$-values; Sheet 2 - mean expression of the average receptor expression level of a cluster and the average ligand expression level of the interacting cluster; Sheet 3 - mean expression of the significant interactions ranked by specificity $(p$-value $<0.05)$. 
bioRxiv preprint doi: https://doi.org/10.1101/467225; this version posted November 9,2018 . The copyright holder for this preprint (which

was not certified by peer review) is the author/funder, who has granted bioRxiv a license to display the preprint in perpetuity. It is made

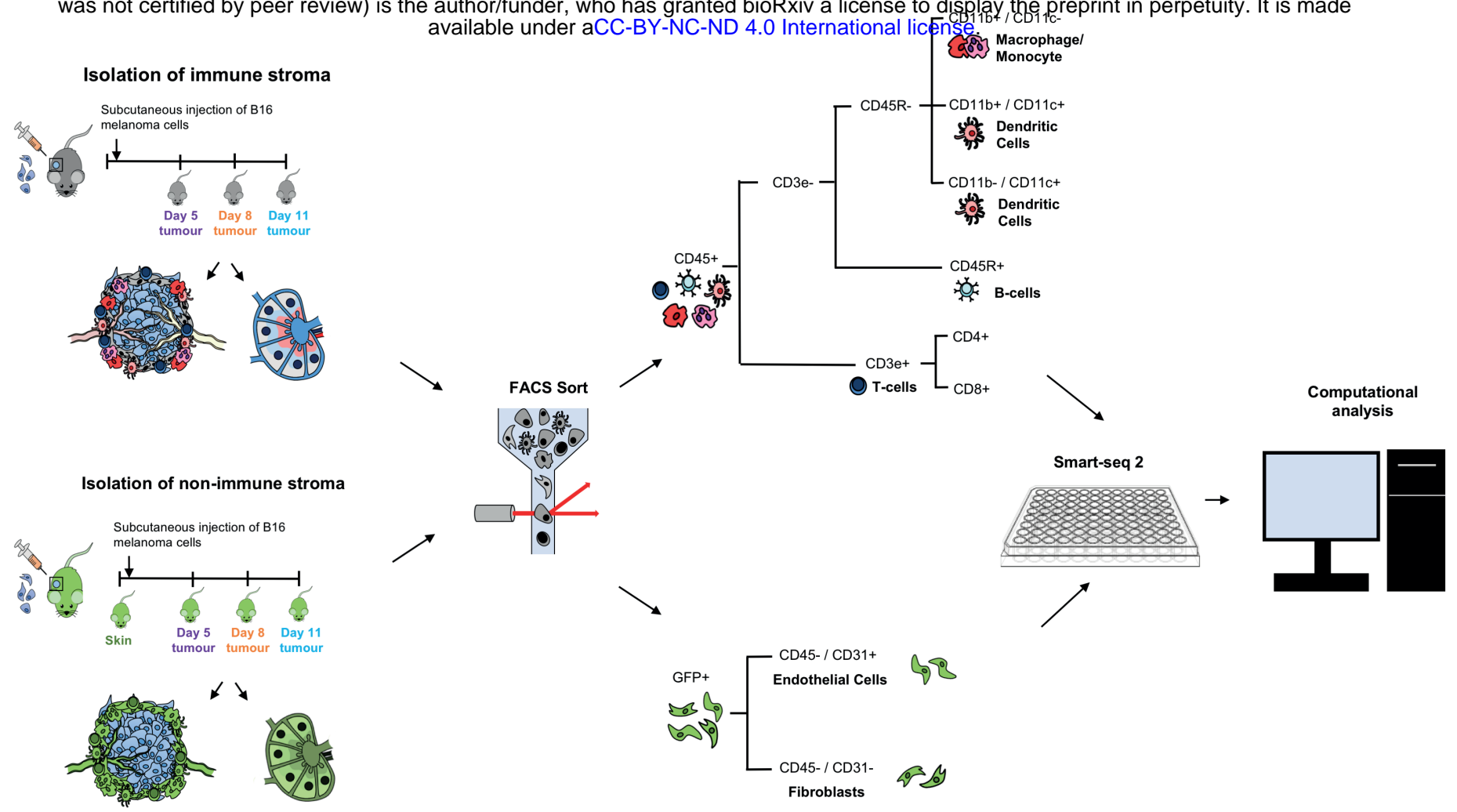

b
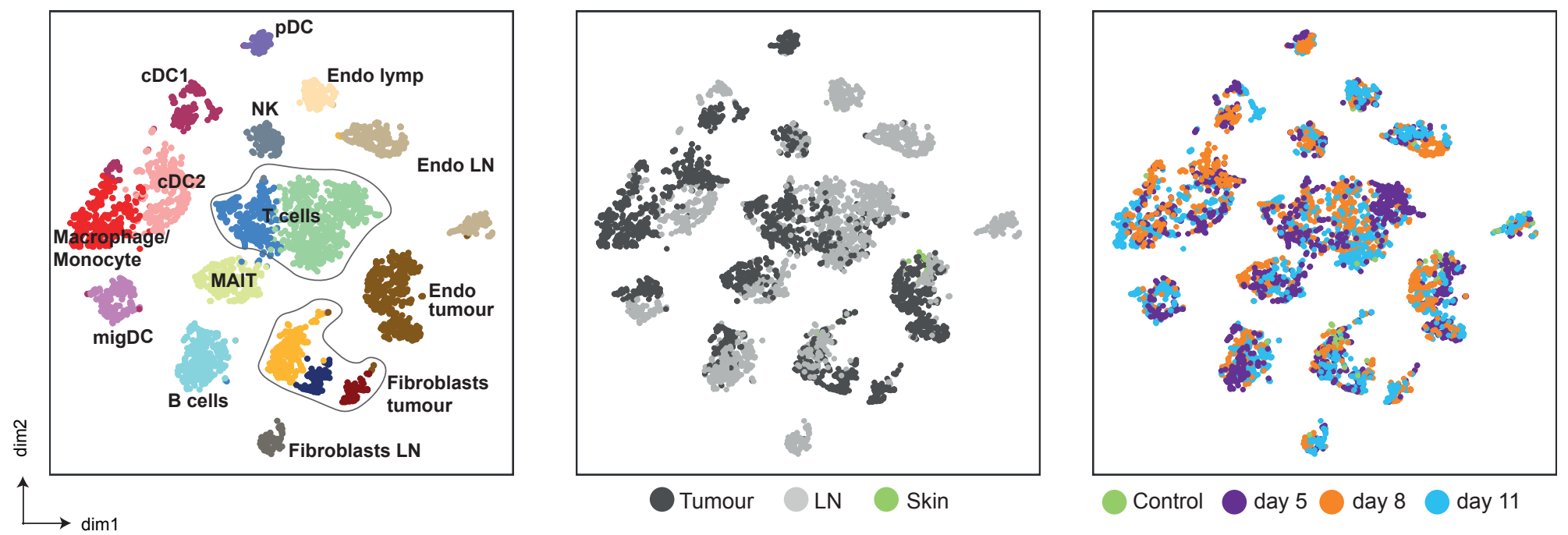

Control day 5 day 8 day 11

C
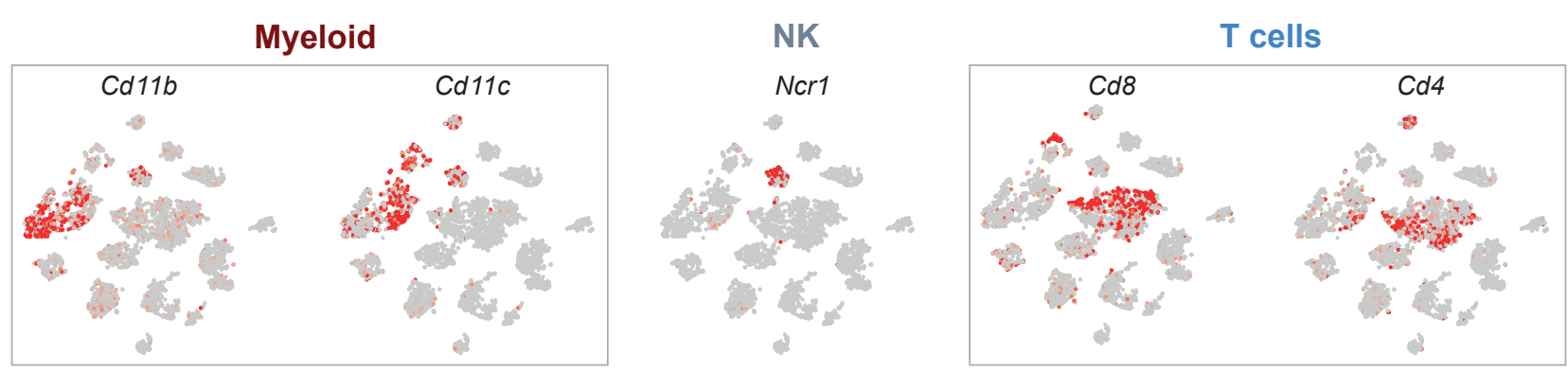

B cells

Fibroblasts

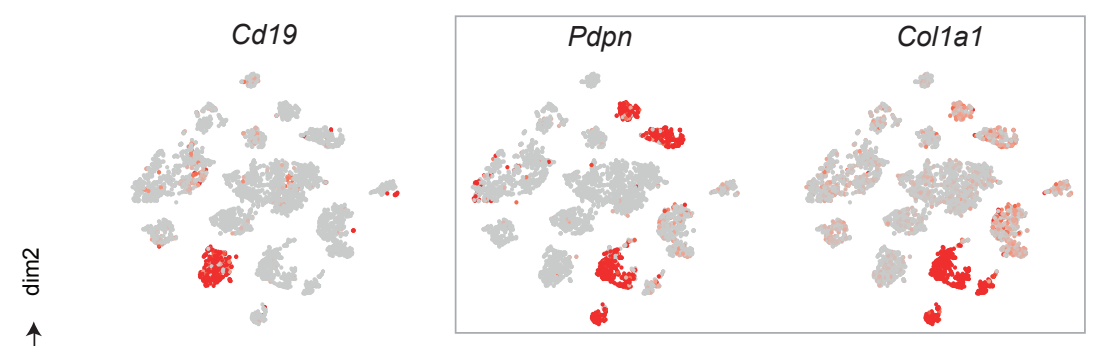

Endothelial

Pecam1
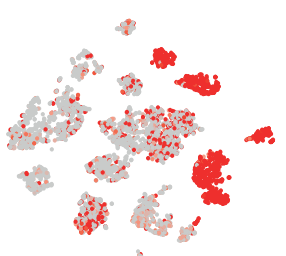

c)
T cells

Endo Lymp

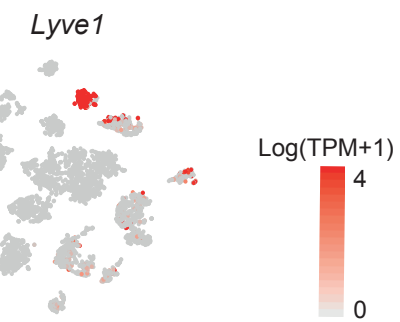


bioRxiv preprint doi: https://doi.org/10.1101/467225; this version posted November 9, 2018. The copyright holder for this preprint (which

was not certified by peer review) is the author/funder, who has granted bioRxiv a license to display the preprint in perpetuity. It is made available under aCC-B b $^{-N C-N D} 4.0$ International license.

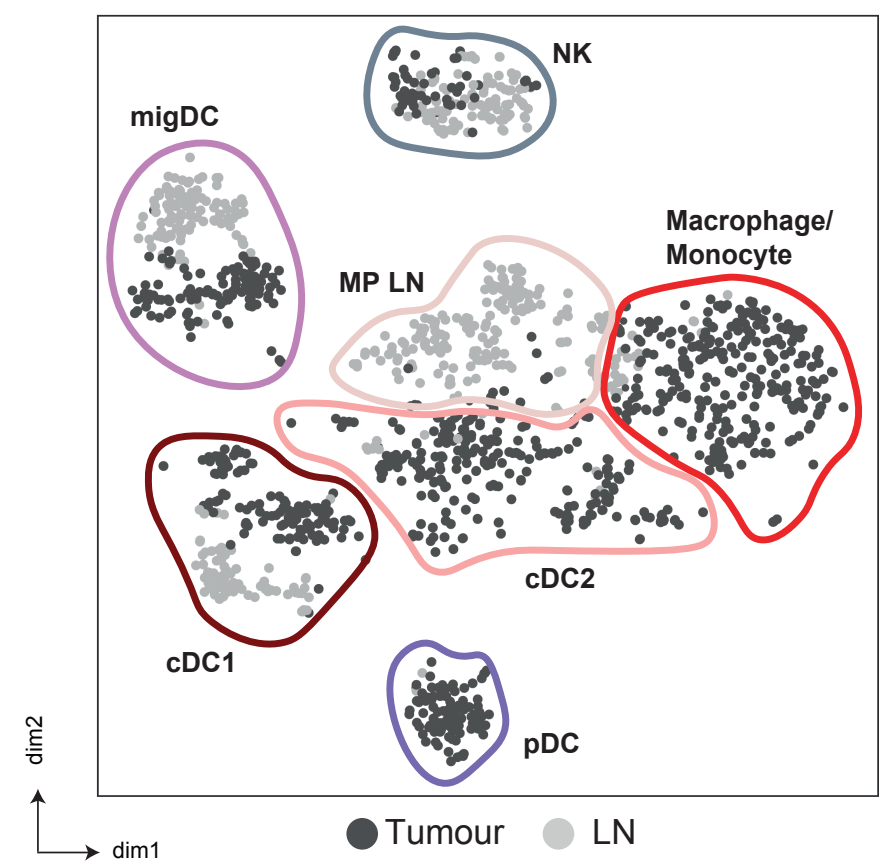

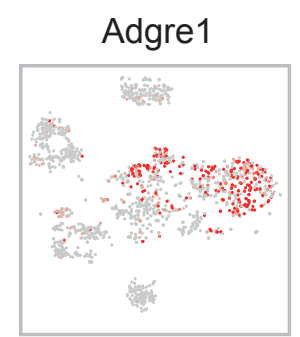
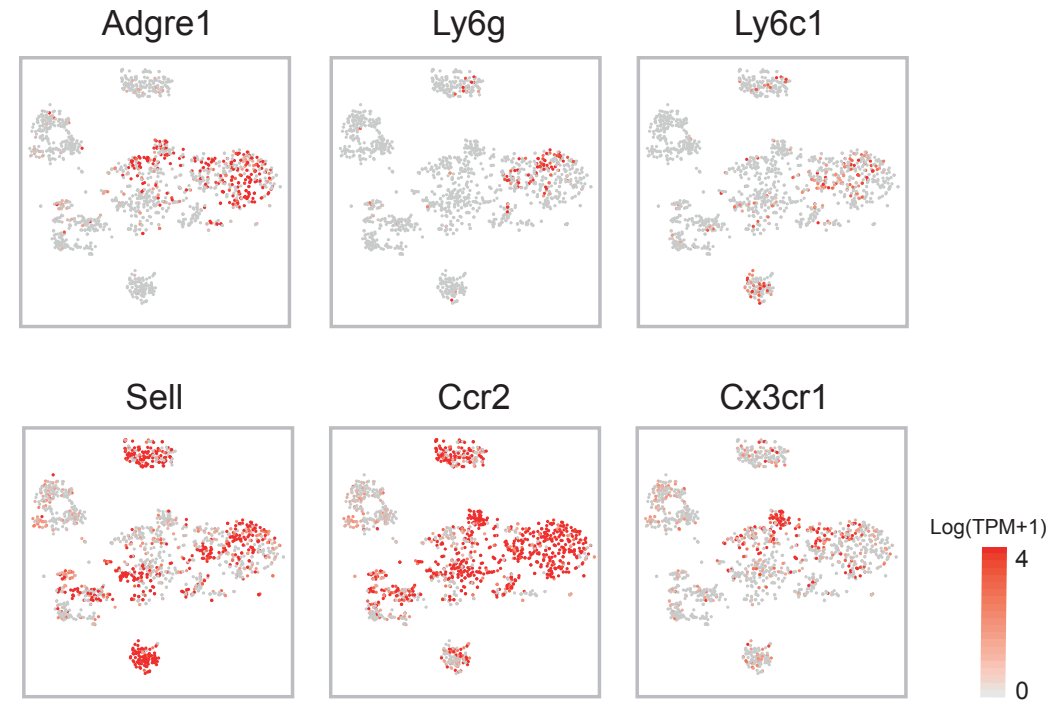

C

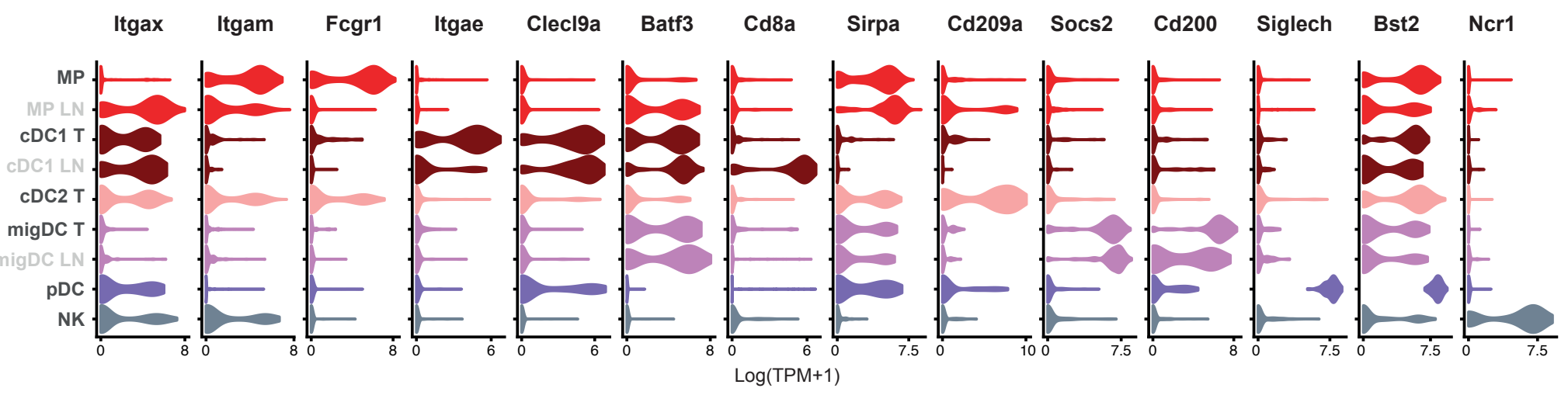

d

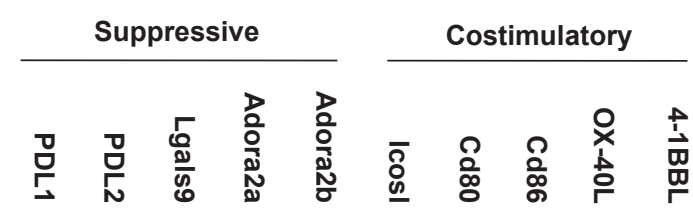
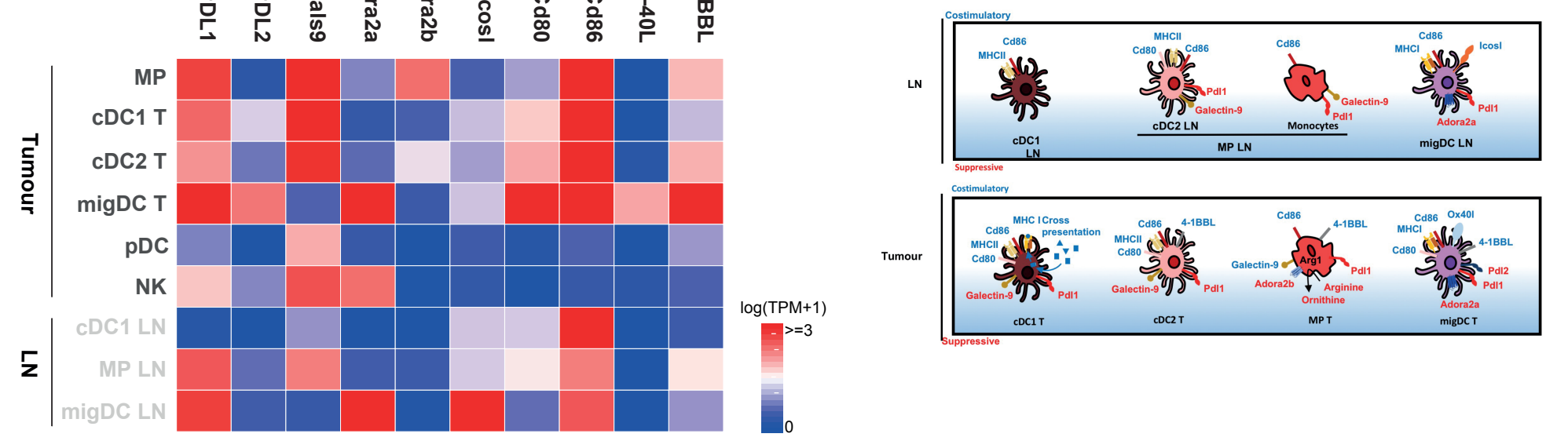

e

\begin{tabular}{|c|c|c|c|c|c|c|c|c|}
\hline \multicolumn{4}{|c|}{ Suppressive } & \multicolumn{5}{|c|}{ Costimulatory } \\
\hline$\underset{\square}{\square}$ & 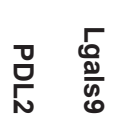 & 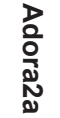 & $\begin{array}{l}\frac{8}{2} \\
\frac{0}{0} \\
\frac{0}{0} \\
\frac{0}{\sigma}\end{array}$ & $\begin{array}{l}\bar{\gamma} \\
\underline{0} \\
\underline{\underline{n}}\end{array}$ & $\frac{0}{\circ}$ & ̊) & $\begin{array}{l}0 \\
\stackrel{x}{0} \\
\hat{0}\end{array}$ & $\begin{array}{l}\stackrel{\vec{i}}{\dot{\Phi}} \\
\text { 离 }\end{array}$ \\
\hline
\end{tabular}

day 5

day 8

day 11 
bioRxiv preprint doi: https://doi.org/10.1101/467225; this version posted November 9, 2018. The copyright holder for this preprint (which was not certified by peer review) is the author/funder, who has granted bioRxiv a license to display the preprint in perpetuity. It is made

a

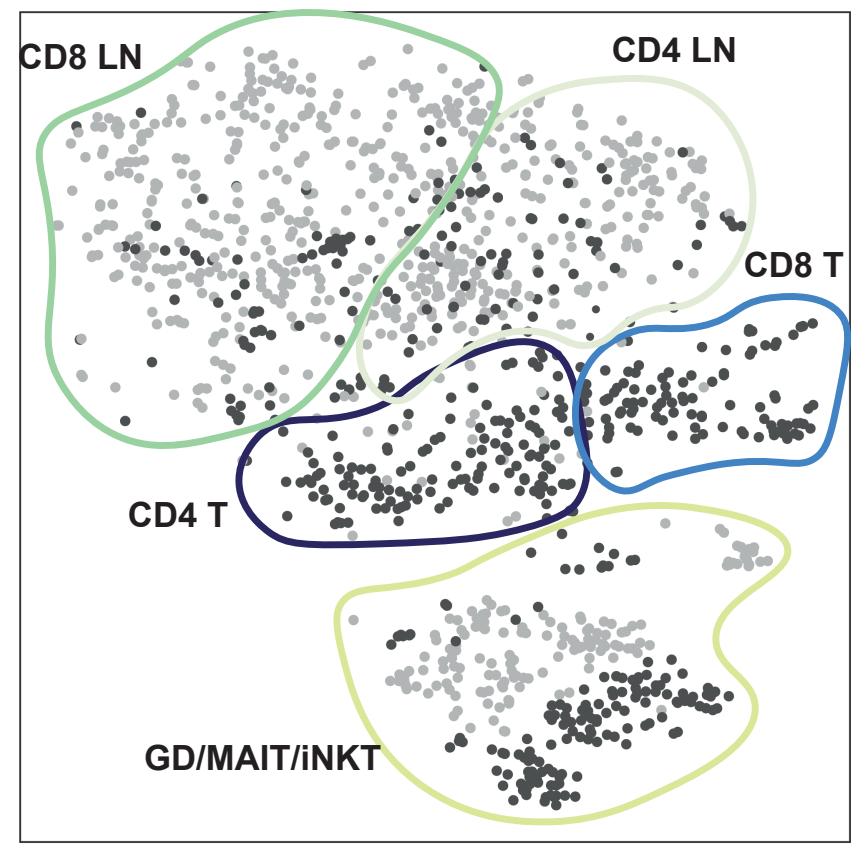

b

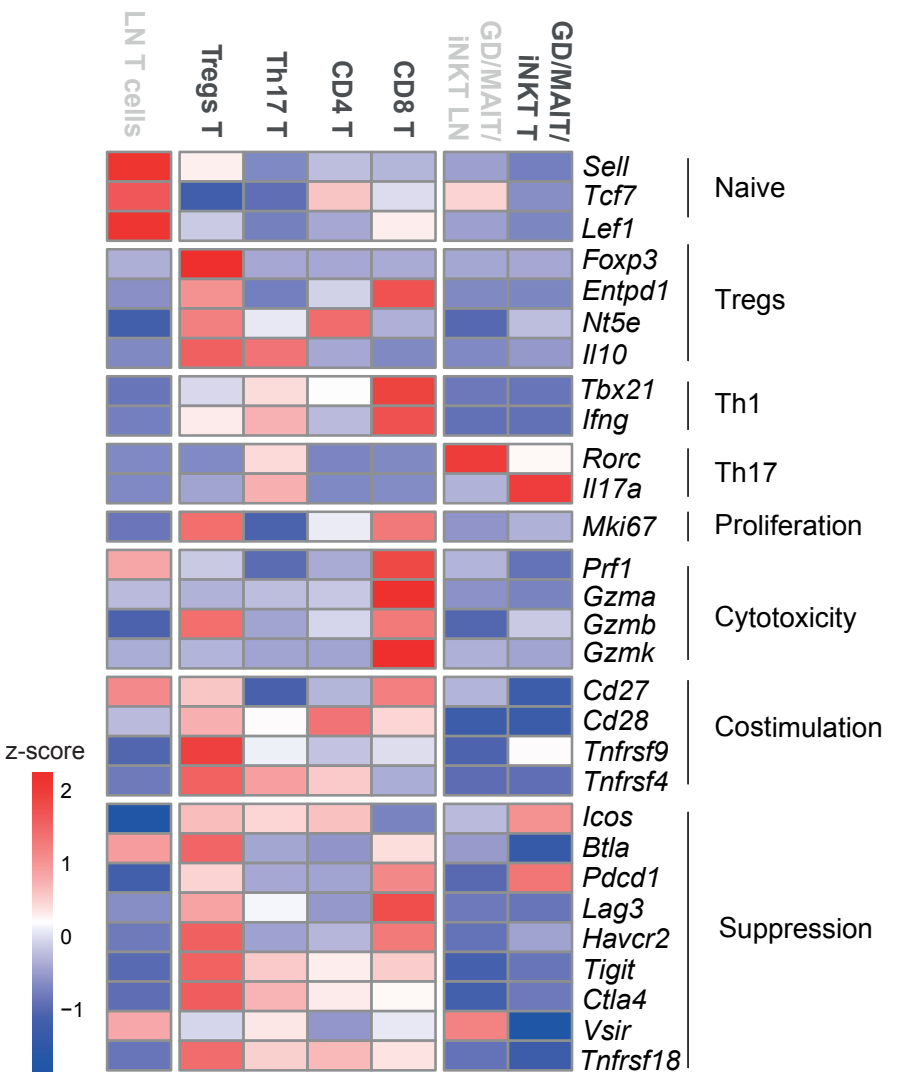

C

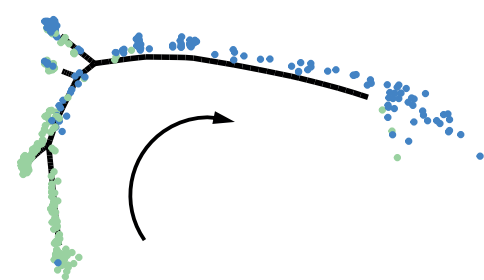

LN Tumour

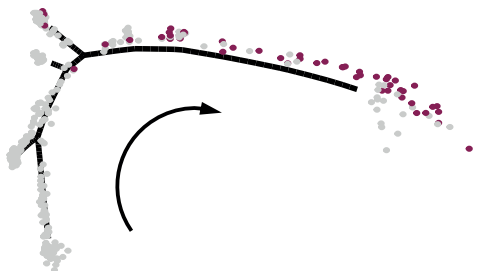

clonally expanded

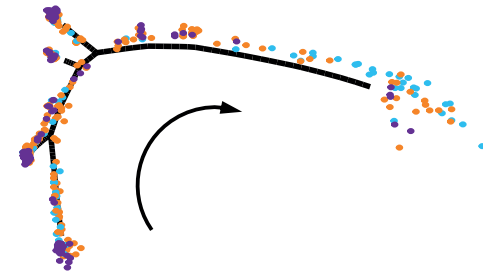

day 5 day 8 day 11

d

Sell

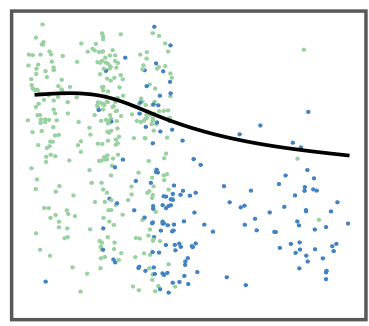

Mki67

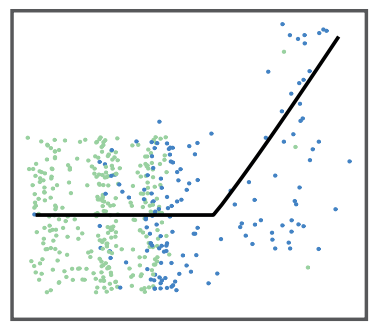

Tcf7

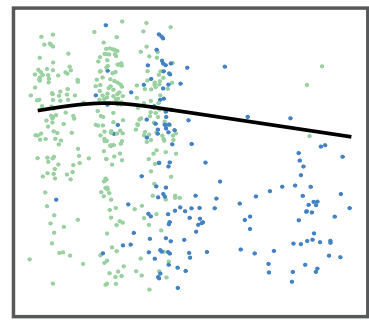

Pdcd1

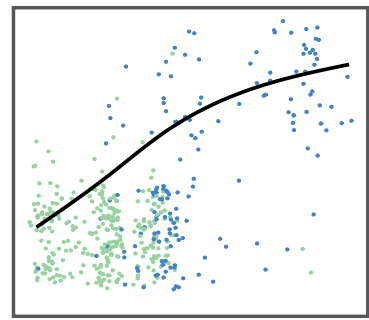

Gzmb

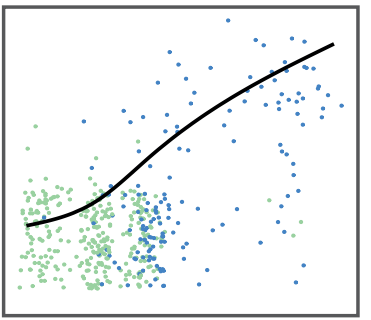

Lag3

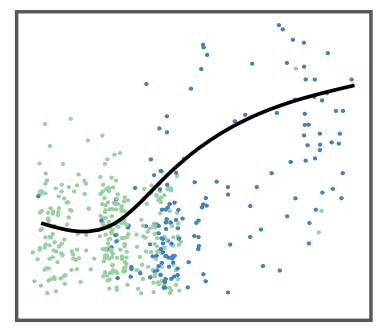

Prf1

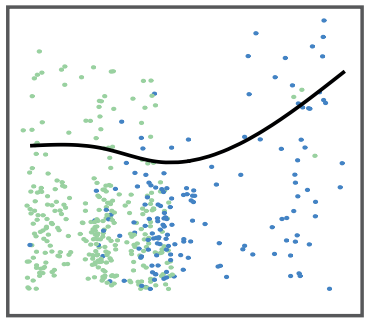

Havcr2

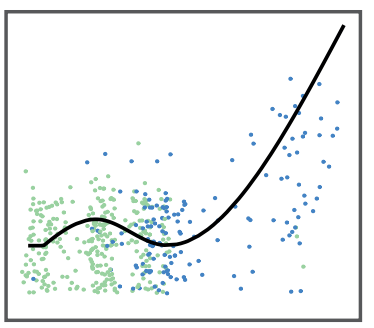


a

bioRxiv preprint doi: https://doi.org/10.1101/467225; this version posted November 9, 2018. The copyright holder for this preprint (which was not certified by peer review) is the author/funder, who has granted bioRxiv a license to dopplay
available under aCC-BY-NC-ND 4.0 International license.

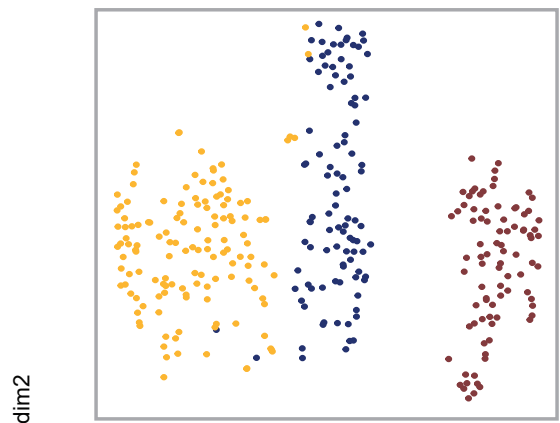

CAF1 CAF2 CAF3 $\operatorname{dim} 1$

C

CAF $1 \square$ CAF $2 \square$ CAF 3

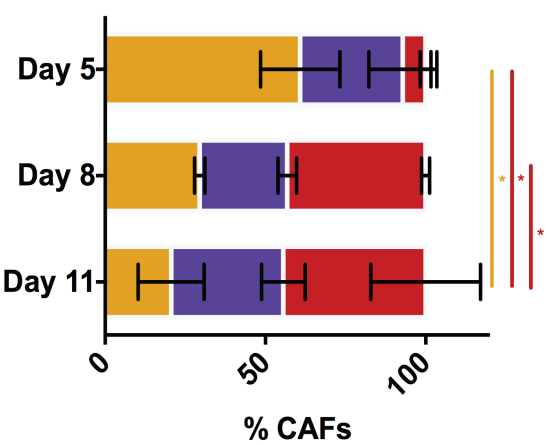

d

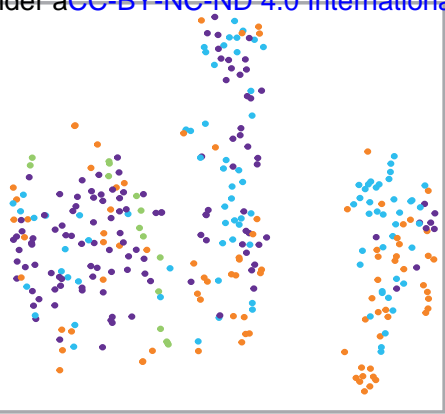

Skin day 5 day 8 day 11

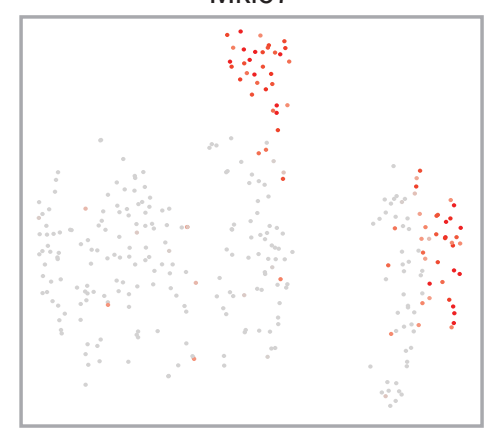

Cytokine Interactions

Complement Cascade

Extra-

Cellular Matrix Interactions

Actin

Cytoskeleton
Cytokine Receptor

$$
\text { 方总量 }
$$

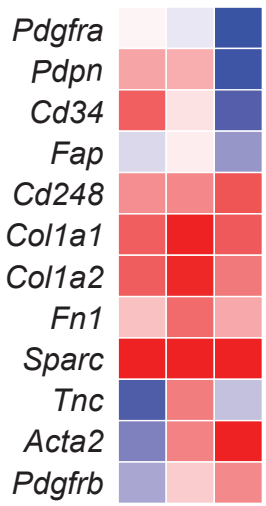

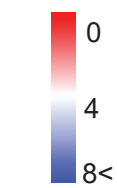

$\log (T P M+1)$ e

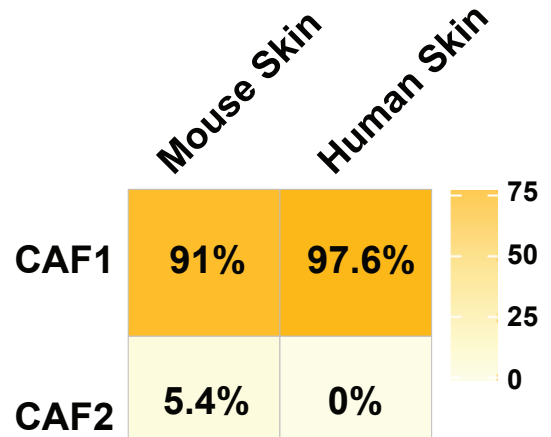

CAF3 $3.6 \%$

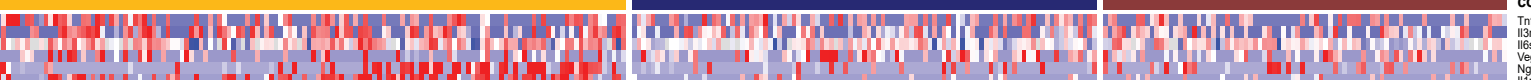

cols z-score

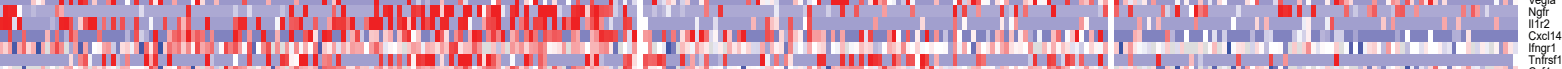

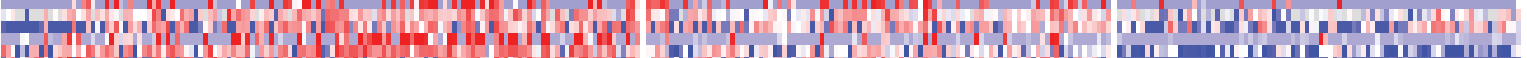

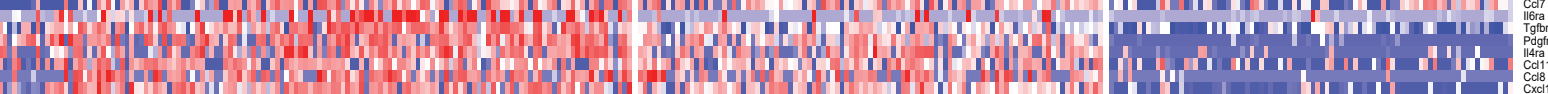

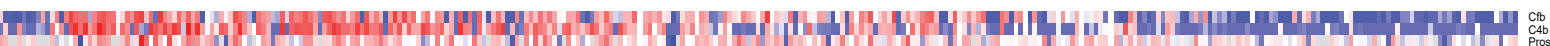
n. ,

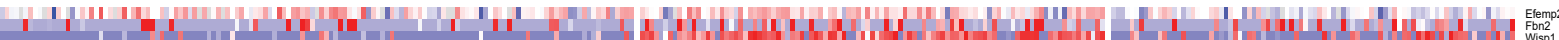

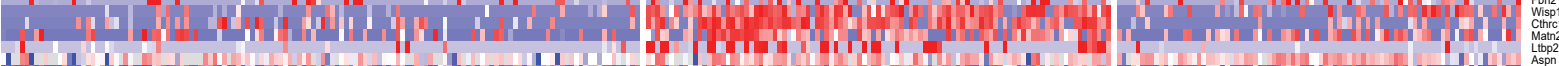

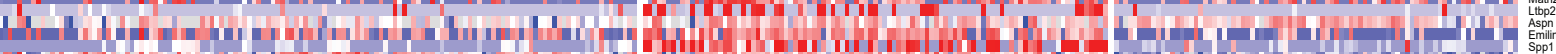

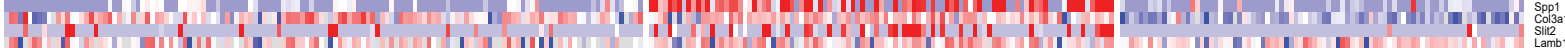

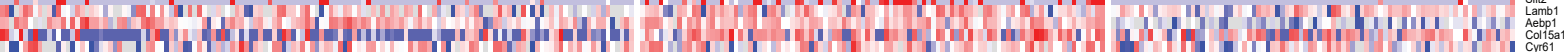

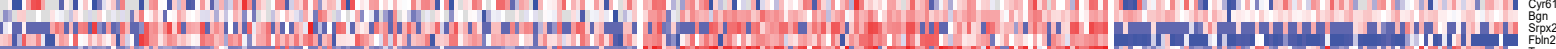
In

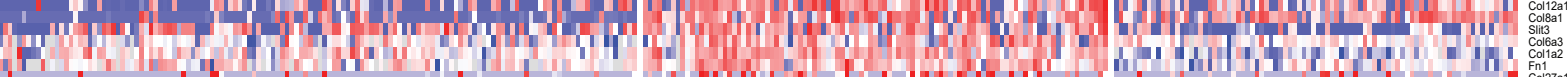

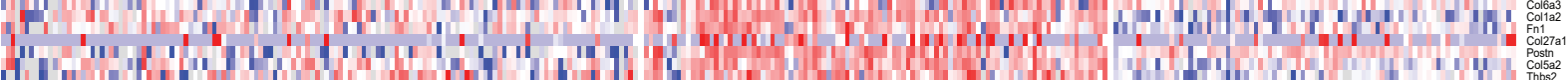

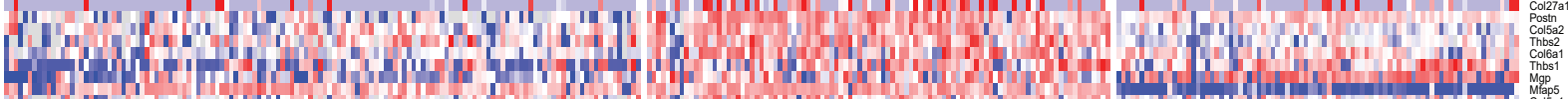

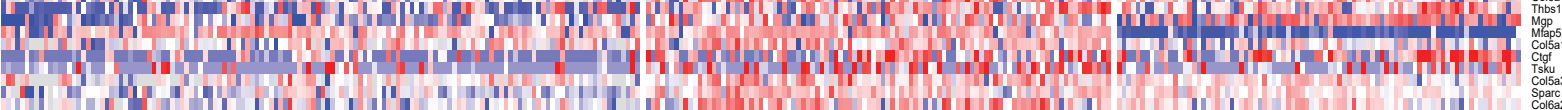

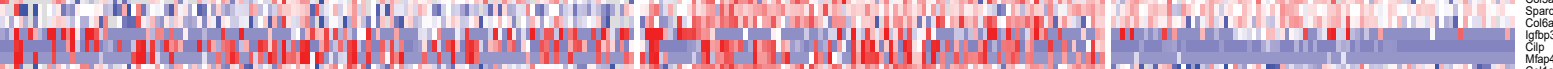
H

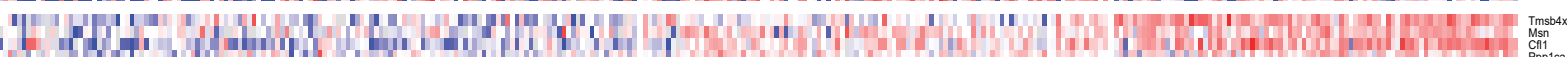

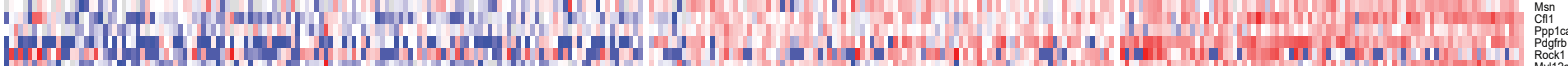
In

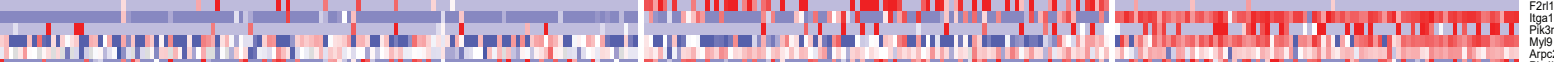

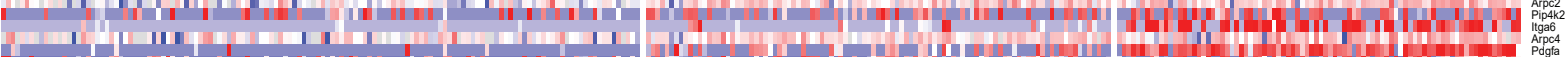

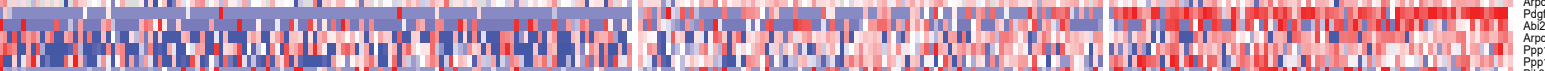
Har

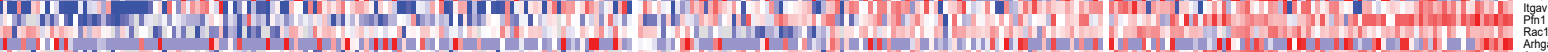

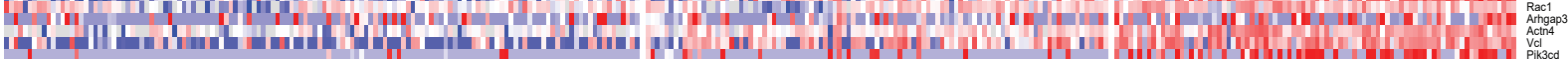

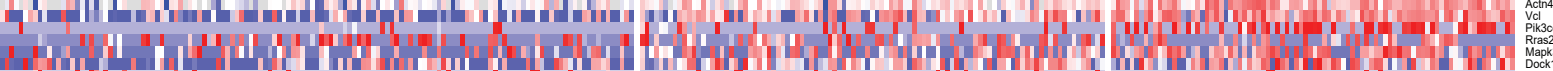

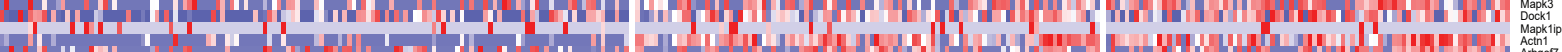

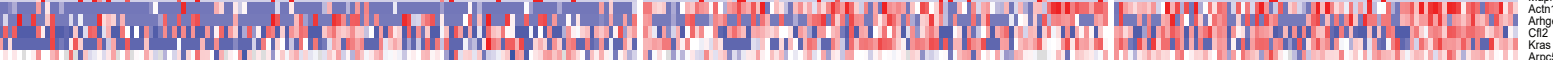

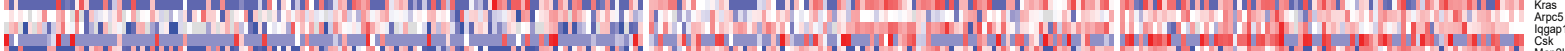

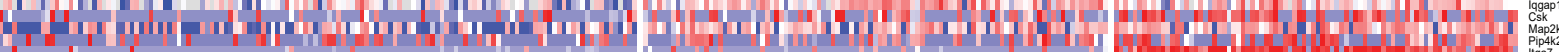

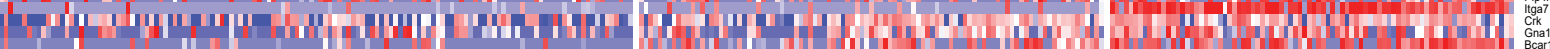


bioRxiv preprint doi: https://doi.org/10.1101/467225; this version posted November 9, 2018. The copyright holder for this preprint (which

was not certified by peer review) is the author/funder, who has granted bioRxiv a license to display the preprint in perpetuity. It is made available under aCC-BY-NC-ND 4.0 International license.

a

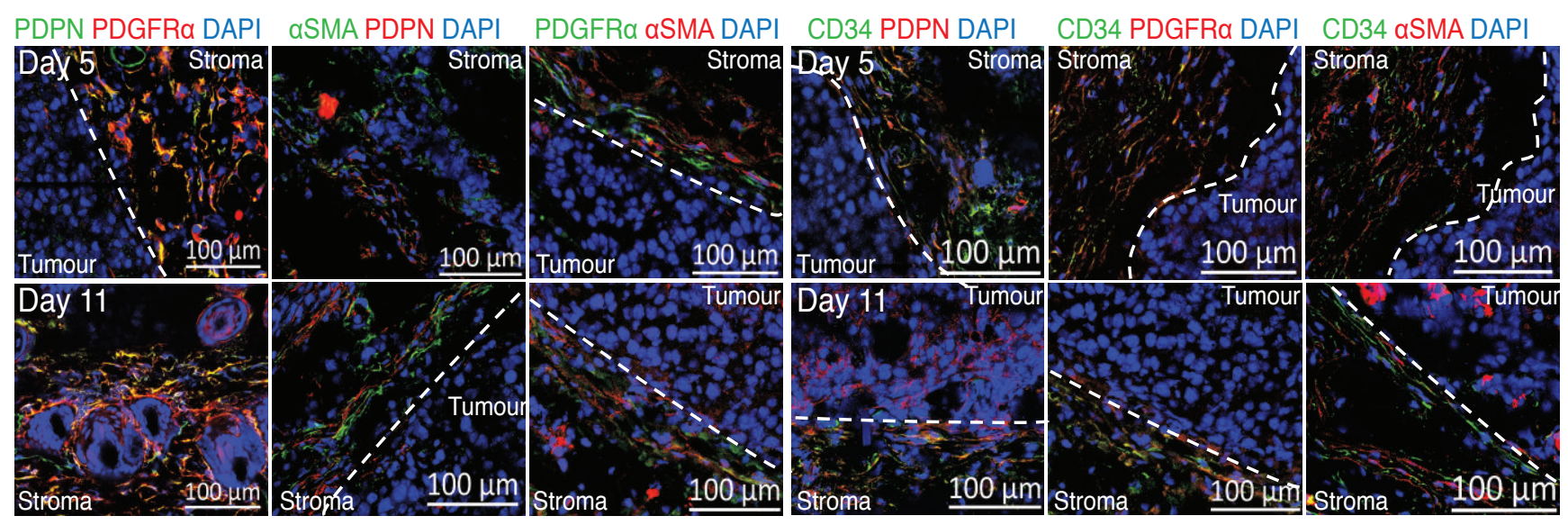

b

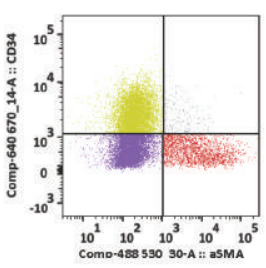

d

CD34 ${ }^{\text {high }}$ aSMA ${ }^{\text {low }}$

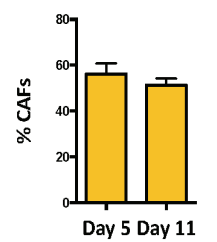

f
C CSF1 CD34 PDGFRa DAPI

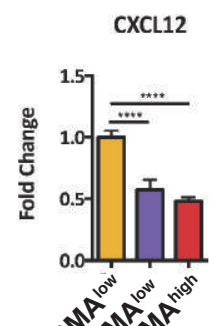

$2 a^{2}$ $0^{300}$

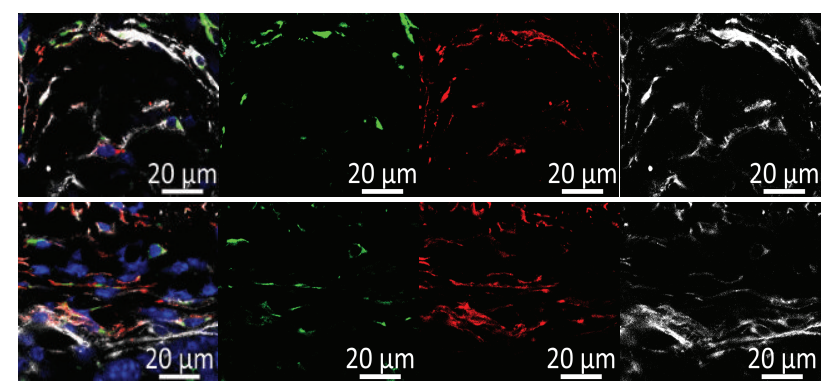

e
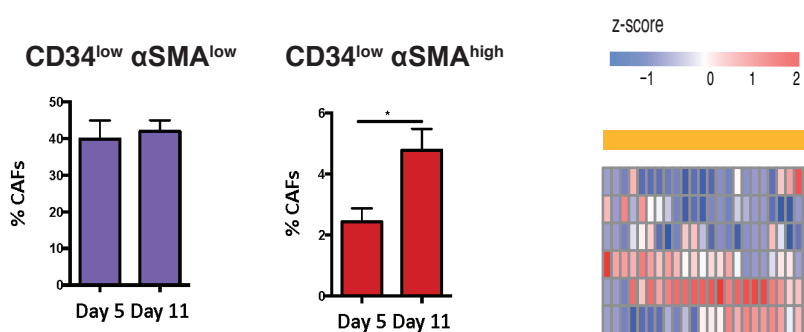

CAF1/2

CAF3

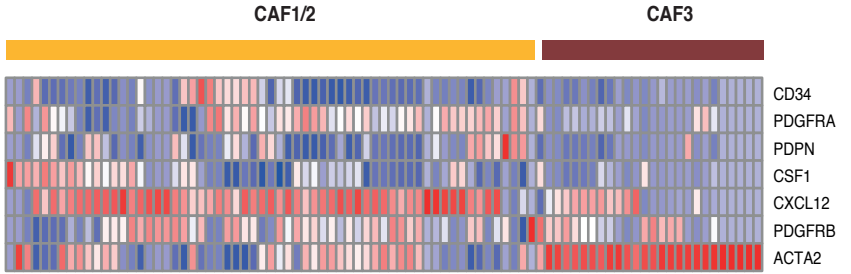

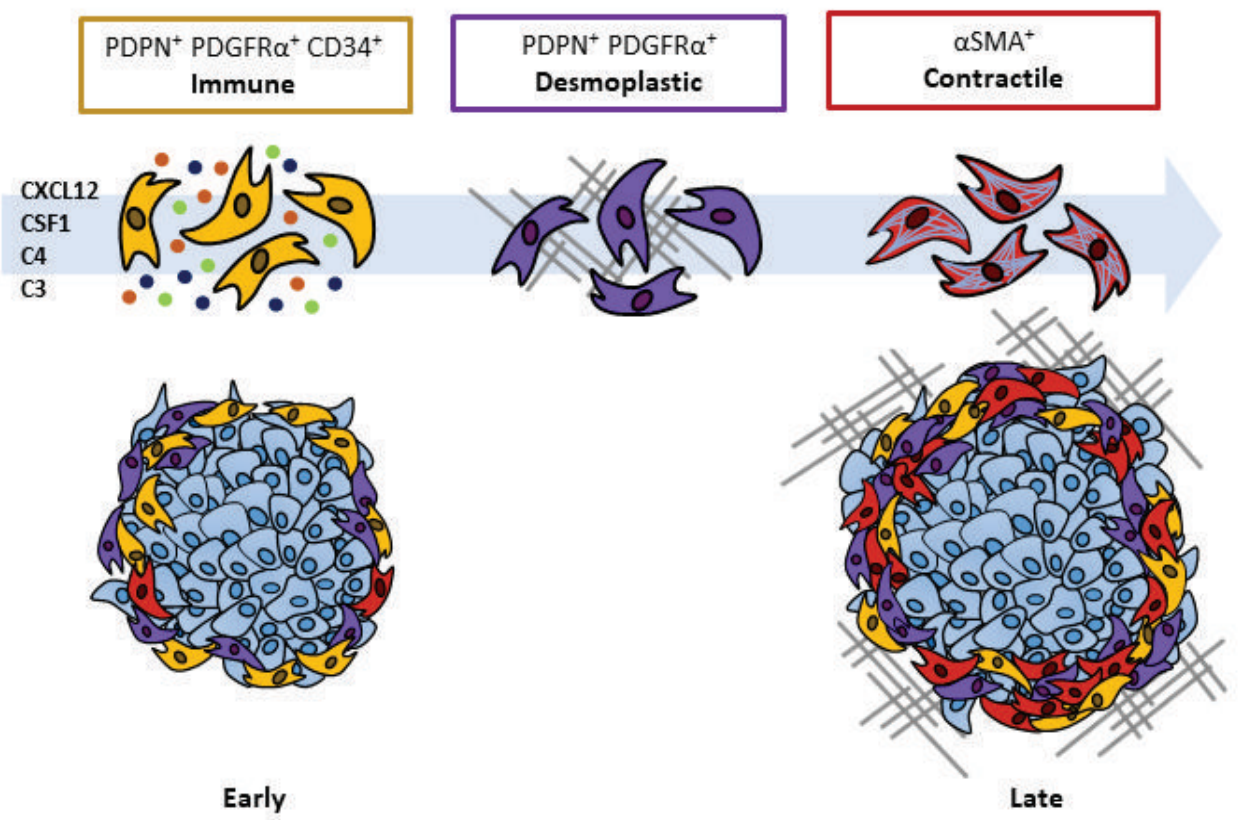


a bioRxiv preprint doi: https://doi.org/10.1101/467225; this version posted November 9,2018 . The copyright holder for this preprint (which

was not certified by peer review) is the author/funder, who has granted bioRxiv a license to display the preprint in perpetuity. It is made available under aCC-BY-NC-ND 4.0 International license.

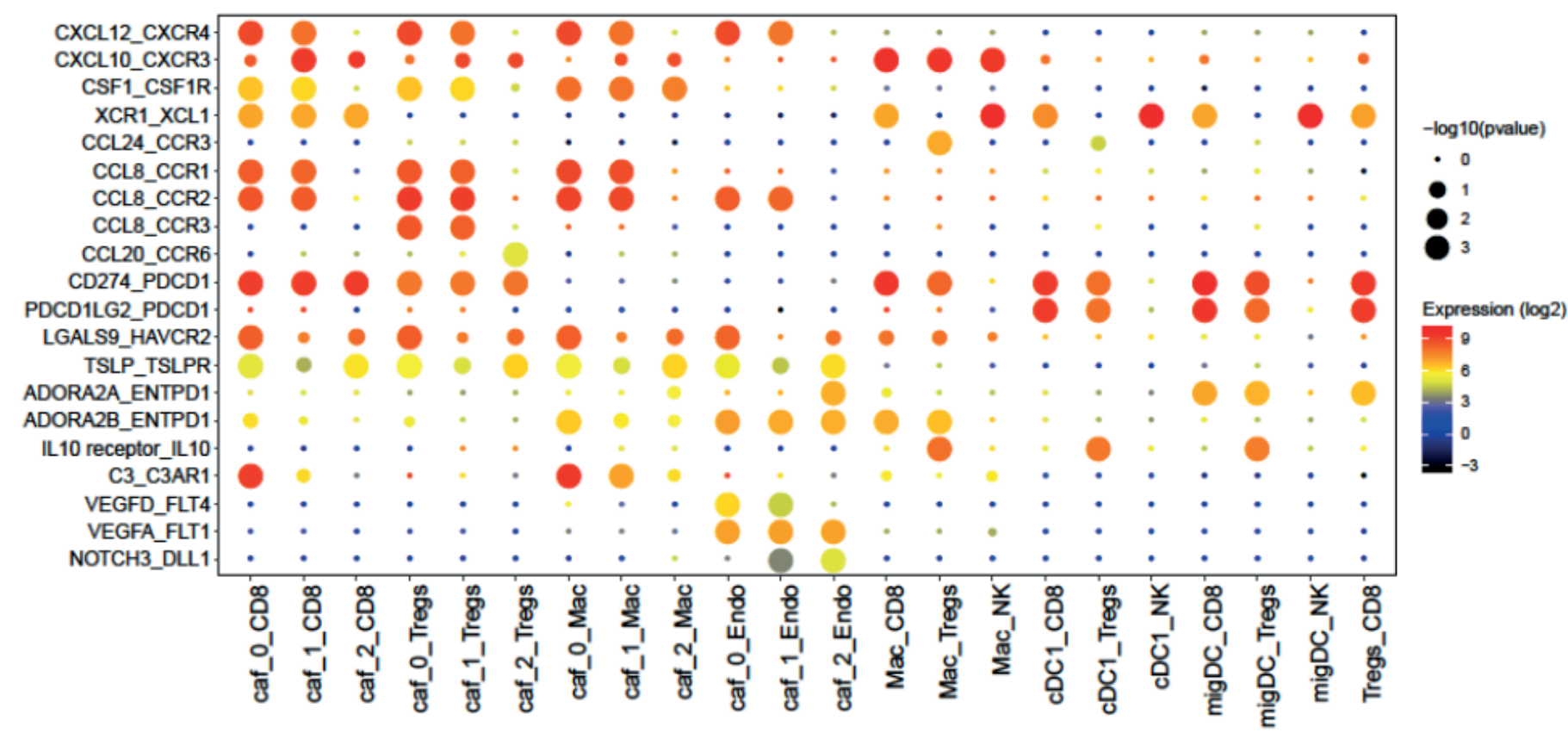

b

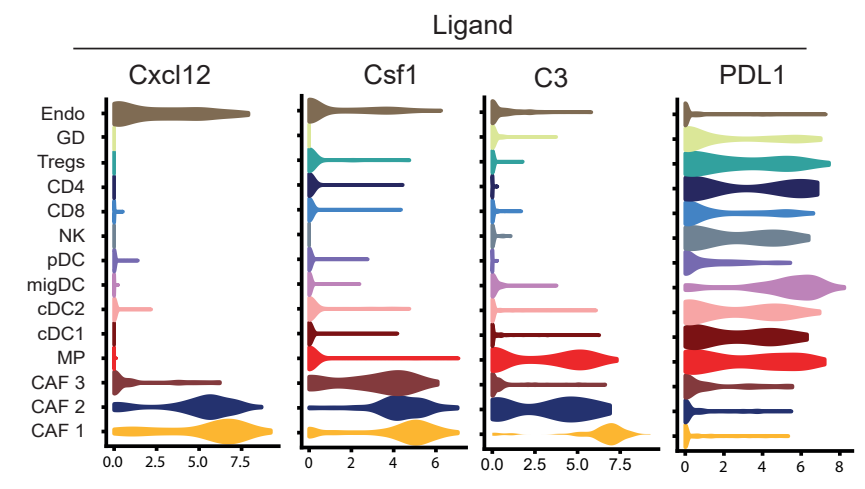

C

\section{F4/80 CSF1R PDPN CD34}
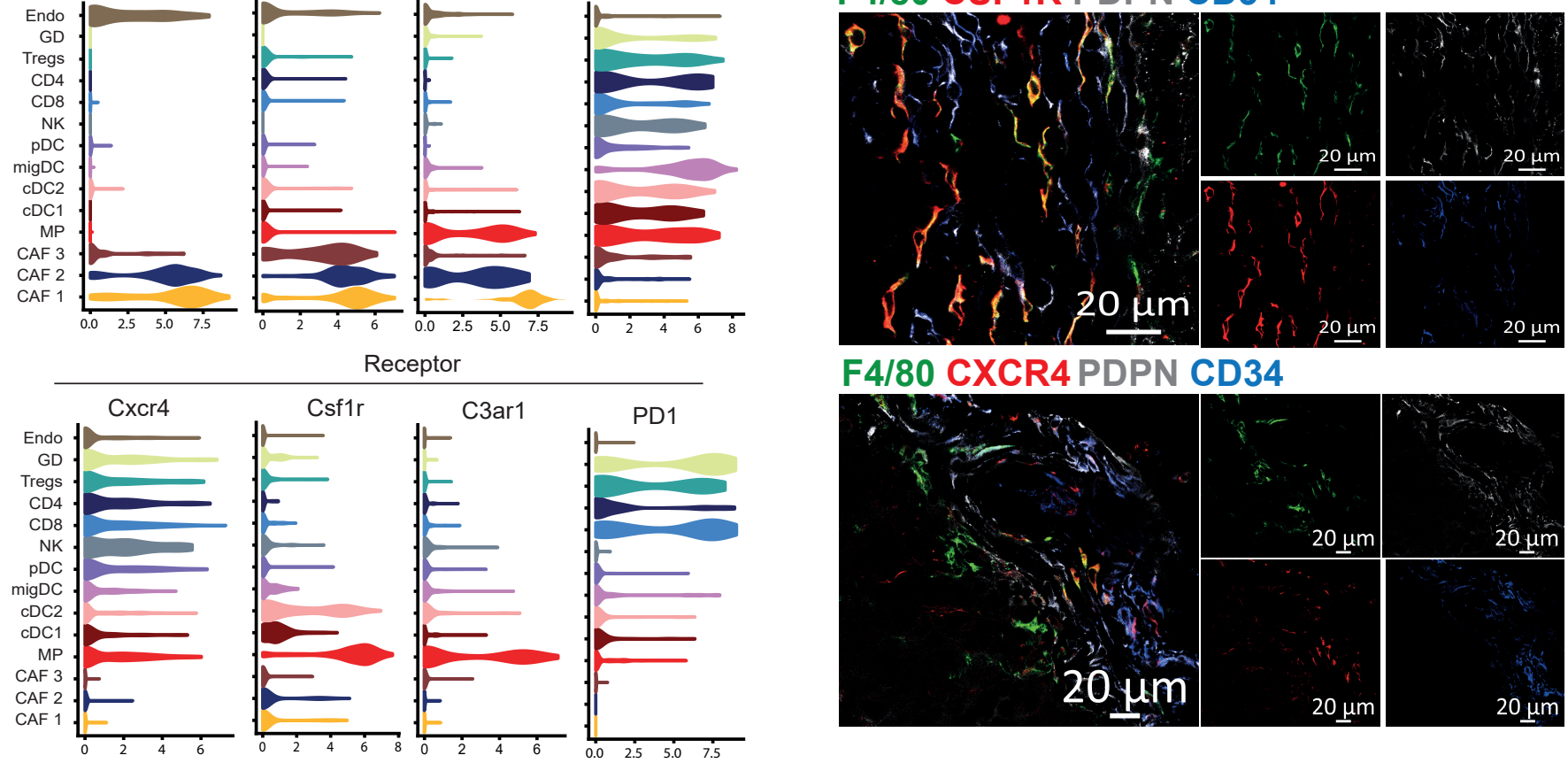

F4/80 CXCR4 PDPN CD34

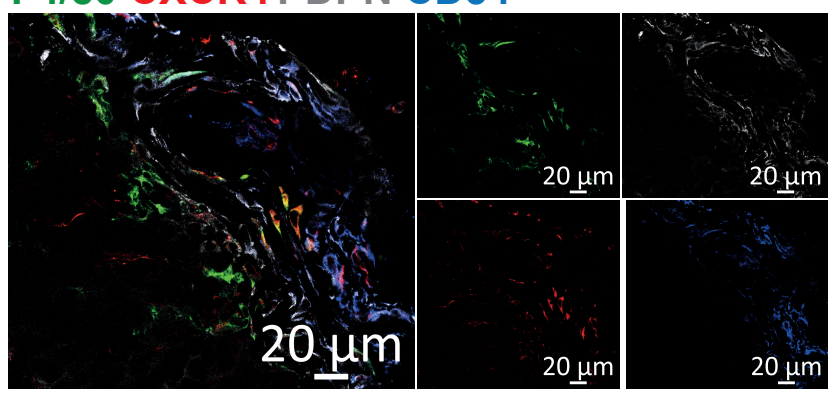

d

Early

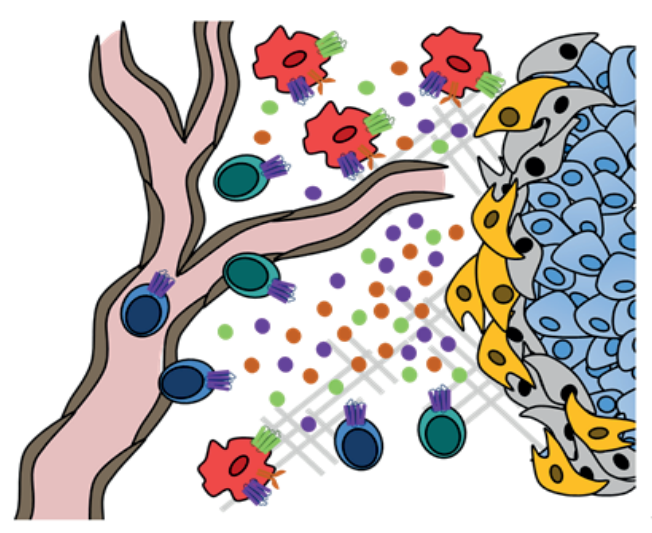

Late

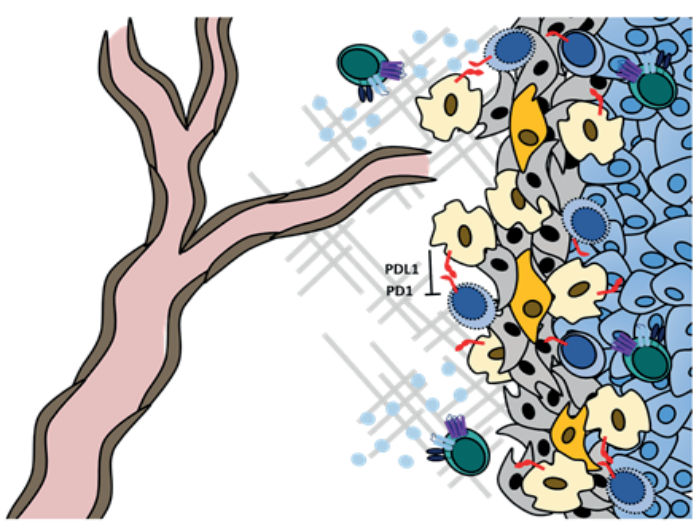

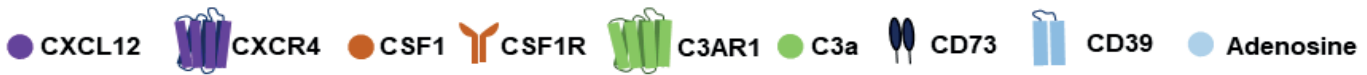

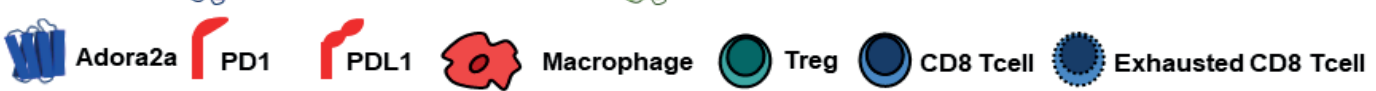

